\title{
MAXIMUM NUMBER OF SUM-FREE COLORINGS IN FINITE ABELIAN GROUPS
}

\author{
HIỆP HÀN AND ANDREA JIMÉNEZ
}

\begin{abstract}
An $r$-coloring of a subset $A$ of a finite abelian group $G$ is called sum-free if it does not induce a monochromatic Schur triple, i.e., a triple of elements $a, b, c \in A$ with $a+b=c$. We investigate $\kappa_{r, G}$, the maximum number of sum-free $r$-colorings admitted by subsets of $G$, and our results show a close relationship between $\kappa_{r, G}$ and largest sum-free sets of $G$.

Given a sufficiently large abelian group $G$ of type $I$, i.e., $|G|$ has a prime divisor $q$ with $q \equiv 2$ ( $\bmod 3)$. For $r=2,3$ we show that a subset $A \subset G$ achieves $\kappa_{r, G}$ if and only if $A$ is a largest sum-free set of $G$. For even order $G$ the result extends to $r=4,5$, where the phenomenon persists only if $G$ has a unique largest sum-free set. On the contrary, if the largest sum-free set in $G$ is not unique then $A$ attains $\kappa_{r, G}$ if and only if it is the union of two largest sum-free sets (in case $r=4$ ) and the union of three ("independent") largest sum-free sets (in case $r=5$ ).

Our approach relies on the so called container method and can be extended to larger $r$ in case $G$ is of even order and contains sufficiently many largest sum-free sets.
\end{abstract}

\section{INTRODUCTION}

A Schur triple in an abelian group $G$ is a triple $(a, b, c)$ with $a+b=c$, and a set $A \subset G$ is sum-free if $A$ contains no such triple. Given a not necessarily sum-free set $A \subset G$, a coloring of the elements of $A$ with $r$ colors is called a sum-free $r$-coloring if each of the color classes is a sumfree set. Sum-free colorings are among the classical objects studied in extremal combinatorics and can be traced back to Schur's theorem [17], one of the first results in Ramsey theory.

In this paper we investigate the maximum number of sum-free colorings admitted by subsets of a given finite abelian group. This is a variant of a problem posed by Erdős and Rothchild [6, 7] for graphs, see Section 1.1. Let $\kappa_{r}(A)$ denote the number of all sum-free $r$-colorings of $A \subset G$ and let the maximum over all $A \subset G$ be denoted by

$$
\kappa_{r, G}=\max \left\{\kappa_{r}(A): A \subset G\right\} .
$$

We are interested in the questions as how large $\kappa_{r, G}$ can be, given $r \geq 2$ and $G$, and which subsets of $G$ achieve the maximum.

A straightforward lower bound for $\kappa_{r, G}$ is obtained by considering a largest sum-free set $B \subset G$, which gives $\kappa_{r, G} \geq r^{|B|}$. The size of largest sum-free sets of $G$, denoted by $\mu(G)$, is

The first author was supported by the FONDECYT Iniciación grant 11150913.

The second author was supported by CONICYT/FONDECYT/POSTDOCTORADO 3150673.

The authors acknowledge the support of Nucleo Milenio Información y Coordinación en Redes ICM/FIC RC130003, Chile. 
a classical and well-understood quantity which depends only on the factorization of $G$. The characterization of $\mu(G)$ distinguishes the following three types.

Definition 1. Let $G$ be a finite abelian group of order $n$. If $n$ has a prime divisor $q$ such that $q \equiv 2(\bmod 3)$, then we say that $G$ is a type I group. In addition, $G$ is called type $I(q)$ if $q$ is the smallest such prime. If $G$ is not of type $I$ and $3 \mid n$ then we say that $G$ is of type II. Otherwise $G$ is called a type III group.

For groups $G$ of type I and type II the quantity $\mu(G)$ was determined by Diananda and Yap [5]. The problem for groups of type III appears to be far more complicated and was only resolved decades later by Green and Ruzsa [9] (see [15, 18, 19] for partial results for type III groups and [3] for the characterization of the largest sum-free sets therein). The results in [5, 9] determine $\mu(G)$ as follows:

$$
\mu(G)= \begin{cases}\left(\frac{1}{3}+\frac{1}{3 q}\right) n & \text { if } G \text { is of type I }(q), \\ \frac{n}{3} & \text { if } G \text { is of type II, } \\ \left(\frac{1}{3}-\frac{1}{m}\right) n & \text { if } G \text { is of type III and } m \text { is the largest order of an element in } G .\end{cases}
$$

For arbitrary abelian groups, we have the following upper bounds which are asymptotically sharp in the exponent for $r=2,3$.

Proposition 2. Given $r \geq 2$ and a finite abelian group $G$ of order $n$, then

$$
\log _{2}\left(\kappa_{2, G}\right) \leq \mu(G)+O\left(n(\log n)^{-\frac{1}{45}}\right)
$$

and for $r \geq 3$

$$
\log _{3}\left(\kappa_{r, G}\right) \leq \frac{r \mu(G)}{3}+O\left(n(\log n)^{-\frac{1}{45}}\right)
$$

Although $\mu(G)$ is known for all finite abelian groups, it is safe to claim that those of type I are much better understood (see Section 3). This additional knowledge allows us to completely resolve the problem for two and three colors in groups of type I of sufficiently large order. In these cases the straightforward lower bound from above is indeed sharp and only the largest sum-free sets achieve the maximum.

Theorem 3. Let $r \in\{2,3\}, q \in \mathbb{N}$ and let $G$ be a type $I(q)$ group of sufficiently large order. Then $\kappa_{r, G}=r^{\mu(G)}$ and $\kappa_{r}(A)=\kappa_{r, G}$ if and only if $A$ is a largest sum-free set in $G$.

For more than three colors this phenomenon does not persist in general and the problem becomes considerably more complicated. We therefore restrict our consideration to type I $(2)$ groups, i.e., those of even order. For these groups our second result resolves the problem for $r=4,5$. Further, we shall see in the course of the paper that our method can be extended to more than five colors. We refer to Section 6 for further discussions.

Before stating the result we note that two largest sum-free sets $B_{1}, B_{2}$ in an abelian group of even order give rise to another through $B_{3}=B_{1} \triangle B_{2}$ (see Corollary 21). In particular, 
$B_{3} \subset B_{1} \cup B_{2}$ holds in this case and there is no even order group with exactly two largest sum-free sets. To distinguish the two possible cases we call a tuple $\left(B_{1}, \ldots, B_{t}\right)$ of largest sumfree sets independent, if none of the $B_{i}$ 's is contained in the union of the remaining ones, or dependent, if the opposite holds.

Theorem 4. Let $G$ be a sufficiently large group of even order. If $G$ contains a unique largest sum-free set then this set and only this set maximizes the number of sum-free $r$-colorings for $r=4,5$. Otherwise

- $A \subset G$ maximizes the number of sum-free 4-colorings if and only if $A$ is the union of two largest sum-free sets.

- $A \subset G$ maximizes the number of sum-free 5-colorings if and only if $A$ is the union of three largest sum-free sets $B_{1}, B_{2}, B_{3}$. Moreover, if $\left(B_{1}, B_{2}, B_{3}\right)$ is independent, then $\kappa_{5}(A)=$ $(1+o(1)) 181440 \cdot 6^{n / 2}$ and if $\left(B_{1}, B_{2}, B_{3}\right)$ is dependent, then $\kappa_{5}(A)=(1+o(1)) 90 \cdot 6^{n / 2}$.

To emphasize the last point of the theorem note that the number of sum-free 5-colorings admitted by an independent triple and that of a dependent one are within one another by a multiplicative constant independent of $n$. In contrast, the stability type Theorem 10 implies that any set which differs from these extremal configurations by $\Omega\left(n(\log n)^{-1 / 27}\right)$ elements admits exponentially fewer sum-free 5-colorings. This phenomenon neither appears for $r=4$ nor for $r=6$ or $r=7$ (see Section 6 ).

1.1. Related results. Problems analogous to the ones considered in this paper were investigated for many other discrete structures (see, e.g., [20, 1, 14, 12, 13, 11, 10]).

Among them the one concerning clique-free edge colorings of graphs is the most prominent one, which moreover seems closest to the problem studied here due to the relationship between triangles and Schur triples].

This problem was raised by Erdős and Rothchild in [6] (see also [7]) and in [20] Yuster showed that, among all graphs on $n$ vertices, only the largest triangle-free graphs maximize the number of triangle-free 2-colorings. Using Szemerédi's regularity lemma Alon, Balogh, Keevash and Sudakov [1] generalized the result to $r=2,3$ colors and cliques $K_{k}$ of size $k \geq 3$. In this case the Turán graphs $T_{k-1}(n)$, i.e., the balanced complete $(k-1)$-partite graphs on $n$ vertices, are the unique graphs which attain the maximum number of $K_{k}$-free $r$-colorings.

Similar to sum-free colorings this phenomenon does not persist for $r>3$ and the question becomes significantly harder. Building on the work of Alon et al., Pikhurko and Yilma [14] determine the unique maximizers for $(r, k)=(4,3)$ and for $(r, k)=(4,4)$. These turn out to be the Turán graphs $T_{4}(n)$ for $k=3$ and $T_{9}(n)$ for $k=4$. For any other pair $(r, k)$, in particular for $r=5$ and $k=3$, the problem remains open.

\footnotetext{
${ }^{1}$ In $\mathbb{F}_{2}^{n}$, for example, consider for given $A \subset \mathbb{F}_{2}^{n}$ the Cayley graph $G_{A}$ which consists of the vertex set $\mathbb{F}_{2}^{n}$ and in which $\{a, b\}$ forms an edge if and only if $a+b \in A$. A triangle $a, b, c$ in $G_{A}$ then corresponds to the Schur triple $a+b, b+c$ and $c+a=c-a$ in $A$.
} 


\section{Outline of the PROOFS AND STABILITy theOREMS}

One possible approach to our problem is to use Green's regularity lemma for abelian groups [8]. In various analogous contexts regularity lemmas have proven to be a suitable tool [1, 14, 12, 13, 11. While this may work well here for groups with many subgroups such as $\mathbb{F}_{p}^{n}$ the technical difficulties are considerable for those lacking subgroups. A novel aspect of our work is to avoid these difficulties by employing the so-called container method. For sum-free sets this comes in the form of a result by Green and Ruzsa [9] which we state in a slightly modified form. We also note that instead of the results of Green and Ruzsa one could also use the container results of Balogh, Morris, Samotij in [4, that of Saxton, Thomason in [16] or a version by Alon et al in [2] which is closely related to that in [4].

Theorem 5 (Proposition 2.1 in [9]). Let $G$ be a finite abelian group of sufficiently large order $n$. For every subset $A \subset G$ there is a family $\mathcal{F}=\mathcal{F}(A)$ of subsets of $A$ (called container family of $A)$ with the following properties

(1) $\log _{2}|\mathcal{F}| \leq n(\log n)^{-1 / 18}$;

(2) Every sum-free set $I \subset A$ is contained in some $F \in \mathcal{F}$;

(3) If $F \in \mathcal{F}$ then $F$ contains at most $n^{2}(\log n)^{-1 / 9}$ Schur triples.

Roughly speaking the theorem states that all sum-free sets in $A$ can be "captured" by a small family of almost sum-free sets. The result of Green and Ruzsa is stated for $A=G$, however, we obviously obtain the family $\mathcal{F}$ as above by taking the intersection of each $F$ with $A$. Here and in what follows the dependence on $A$ is regularly suppressed as it is clear from the context.

With Theorem 5 as the starting point we make the following simple but crucial observation.

Observation 6. Let $\mathcal{F}=\mathcal{F}(A)$ be a container family as in Theorem 5 and let $\Phi_{r}(A)$ denote the set of all sum-free $r$-colorings of $A$. To each $\varphi \in \Phi_{r}(A)$ assign a tuple $\left(F_{1}, \ldots, F_{r}\right) \in \mathcal{F}^{r}$ such that $\varphi^{-1}(i) \subseteq F_{i}$ for every $i \in[r]$ and let $\Phi\left(F_{1}, \ldots, F_{r}\right)$ denote the set of all $\varphi \in \Phi(A)$ assigned to $\left(F_{1}, \ldots, F_{r}\right)$. Note that this assignment is possible due to (2) of Theorem 5 and we have

$$
\Phi_{r}(A)=\bigcup_{\left(F_{1}, \ldots, F_{r}\right) \in \mathcal{F}^{r}} \Phi\left(F_{1}, \ldots, F_{r}\right) .
$$

Further, let $n_{k}$ denote the number of elements in $F_{1} \cup \cdots \cup F_{r}=A$ which are contained in exactly $k$ sets of $\left(F_{1}, \ldots, F_{r}\right)$. Then

$$
\left|\Phi\left(F_{1}, \ldots, F_{r}\right)\right| \leq \prod_{k \in[r]} k^{n_{k}} \quad \text { and } \quad \sum_{k \in[r]} k \cdot n_{k}=\sum_{k \in[r]}\left|F_{k}\right| .
$$

As the number of $r$-tuples $\left(F_{1}, \ldots, F_{r}\right)$ is at most $|\mathcal{F}|^{r} \leq 2^{r n(\log n)^{-1 / 18}} \ll r^{\mu(G)} \leq \kappa_{r, G}$, a set $A$ which admits about as many sum-free $r$-colorings as $\kappa_{r, G}$ must give rise to a "substantial" $r$-tuple $\left(F_{1}, \ldots, F_{r}\right)$, i.e., one for which $\left|\Phi\left(F_{1}, \ldots, F_{r}\right)\right|$ is about as large as $\kappa_{r, G}$. This information will be used to derive stability type results which form an important step in the proofs. The case $r=2,3$ reads as follows. 
Theorem 7. Suppose that $r \in\{2,3\}, q \in \mathbb{N}$ and $0<\varepsilon<\frac{1}{(q+1)}$. Let $G$ be a type $I(q)$ group of sufficiently large order $n$ and let $A \subset G$ be such that $\kappa_{r}(A)>r^{\mu(G)-\frac{\varepsilon n}{200}}$. Then there is a largest sum-free set $B \subset G$ such that $|A \backslash B|<\varepsilon n$.

For $r=4,5$ and even order groups we will need a stronger notion of stability which is harder to establish. One reason for the complication is that in these cases the straightforward lower bound $\kappa_{r, G} \geq r^{\mu(G)}$ is far from best possible, as shown by the following.

Proposition 8. Given an abelian group $G$ of even order with at least two largest sum-free sets and let $B_{1}, B_{2}, B_{3}$ be largest sum-free sets in $G$ with $\left|\left\{B_{1}, B_{2}, B_{3}\right\}\right| \geq 2$. Then

$$
\kappa_{4}\left(B_{1} \cup B_{2}\right) \geq(3 \sqrt{2})^{n / 2} \quad \text { and } \quad \kappa_{5}\left(B_{1} \cup B_{2} \cup B_{3}\right) \geq 6^{n / 2} .
$$

Proposition 8 will be proven at the end of Section 3 . We continue with the description of the structure of "substantial" tuples, i.e., those $\left(F_{1}, \ldots, F_{r}\right)$ such that $\left|\Phi\left(F_{1}, \ldots, F_{r}\right)\right|$ is about as large as the bounds from above. The statement of the results requires some notation.

Let $G$ be a finite abelian group of even order and let $\mathcal{B}=\left(B_{1}, \ldots, B_{t}\right)$ be an ordered tuple of not necessarily distinct largest sum-free sets of $G$. For a largest sum-free set $B$ let $B^{1}=B$ and let $B^{0}=G \backslash B$ be its complement. For an $\varepsilon=\left(\varepsilon_{1}, \ldots, \varepsilon_{t}\right) \in\{0,1\}^{t}$ define the atom $\mathcal{B}(\varepsilon)$ via

$$
\mathcal{B}(\varepsilon)=\bigcap_{i \in[t]} B_{i}^{\varepsilon_{i}} .
$$

If $\sum_{i \in[t]} \varepsilon_{i}=k$ then $\mathcal{B}(\varepsilon)$ is referred to as a $k$-atom. Hence, the elements in a $k$-atom are contained in exactly $k$ of the $B_{i}$ 's. Finally, we say that $\mathcal{B}=\left(B_{1}, \ldots, B_{t}\right)$ consists of a collection of certain atoms if these atoms partition $\cup_{i \in[t]} B_{i}$.

For four colors the intersection structure of "substantial" tuples are identified as follows.

Theorem 9. Given an abelian group $G$ of sufficiently large even order $n$. Let $A \subseteq G$ and let $\mathcal{F}=\mathcal{F}(A)$ be a container family as in Theorem 5. If $B$ is the unique largest sum-free set in $G$ and $A$ satisfies $\kappa_{4}(A)>3.999^{n / 2}$, then $|A \backslash B|<12 n(\log n)^{-1 / 27}$.

If $G$ has at least two largest sum-free sets and the tuple $\left(F_{1}, \ldots, F_{4}\right) \in \mathcal{F}^{4}$ satisfies

$$
\left|\Phi\left(F_{1}, \ldots, F_{4}\right)\right|>\left(3 \sqrt{2}-\frac{1}{25}\right)^{n / 2},
$$

then there exist three largest sum-free sets $B_{1}, B_{2}$ and $B_{3}=B_{1} \triangle B_{2}$ and a function $f:[4] \rightarrow[3]$ such that the following holds

- $\left|F_{i} \backslash B_{f(i)}\right|<3 n(\log n)^{-1 / 27}$ for all $i \in[4]$, and

- $\left(B_{f(1)}, \ldots, B_{f(4)}\right)$ consists of one 2 -atom, and two 3-atoms.

To formulate the corresponding result for five colors, we first note that for an independent triple of largest sum-free sets $\left(B_{1}, B_{2}, B_{3}\right)$, there are seven largest sum-free sets contained in $B_{1} \cup B_{2} \cup B_{3}$. This will be shown in Section 3 (see Corollary [21). The structure of substantial tuples for five colors then reads as follows. 
Theorem 10. Given an abelian group $G$ of sufficiently large even order $n$. Let $A \subseteq G$ and let $\mathcal{F}$ be a container family of $A$ as in Theorem 5 . If $B$ is the unique largest sum-free set in $G$, and A satisfies $\kappa_{5}(A)>4.999^{n / 2}$, then $|A \backslash B|<15 n(\log n)^{-1 / 27}$.

If $G$ has at least two largest sum-free sets and the tuple $\left(F_{1}, \ldots, F_{5}\right) \in \mathcal{F}^{5}$ satisfies

$$
\left|\Phi\left(F_{1}, \ldots, F_{5}\right)\right|>5.9^{n / 2},
$$

then there are three largest sum-free sets $B_{1}, B_{2}, B_{3}$ of $G$ such that one of the following holds.

(1) $B_{3}=B_{1} \triangle B_{2}$ and there is a function $f:[5] \rightarrow$ [3] such that

- $\left|F_{i} \backslash B_{f(i)}\right|<3 n(\log n)^{-1 / 27}$ for all $i \in[5]$ and

- $\left(B_{f(1)}, \ldots, B_{f(5)}\right)$ consists of one 4-atom, and two 3-atoms.

(2) There are four distinct largest sum-free sets $B_{4}, \ldots, B_{7}$ contained in $B_{1} \cup B_{2} \cup B_{3}$ and a function $f:[5] \rightarrow[7]$ such that

- $\left|F_{i} \backslash B_{f(i)}\right|<3 n(\log n)^{-1 / 27}$ for all $i \in[5]$ and

- $\left(B_{f(1)}, \ldots, B_{f(5)}\right)$ consists of two 2-atoms, four 3-atoms, and one 4-atom.

Our approach to Theorem 9 and Theorem 10 is to reduce the problems we want to solve for general abelian even order groups to a related problem in $\mathbb{F}_{2}^{t}$ for some $t \leq r \leq 5$. This reduction and the proof of Theorem 7 will require further group theoretic facts which we introduce in the next section.

\section{Group theoretic FaCts}

The size and the structure of largest sum-free sets in type I abelian groups were determined by Diananda and Yap as follows.

Theorem 11 (Diananda and Yap [5]). Let $G$ be a type $I(q)$ group of order $n$. Then the size of the largest sum-free set $\mu(G)$ satisfies $\mu(G)=\left(\frac{1}{3}+\frac{1}{3 q}\right) n$. Moreover, if $B$ is a largest sumfree set in $G$, then $B$ is a union of cosets of some subgroup $H$ of order $n / q$ of $G, B / H$ is in arithmetic progression and $B \cup(B+B)=G$.

The following results are due to Green and Ruzsa [9].

Lemma 12 (Proposition 2.2 in [9]). If $G$ is abelian and $A \subset G$ contains at most $\delta n^{2}$ distinct Schur triples, then $|A| \leq \mu(G)+2^{20} \delta^{1 / 5} n$.

Lemma 13 (Lemma 4.2 in [9]). Let $\varepsilon>0$ and let $G$ be an abelian group. If $A \subseteq G$ has size at least $n / 3+\varepsilon n$ and contains at most $\varepsilon^{3} n^{2} / 27$ distinct sums, then there is a sum-free set $S \subseteq A$ such that $|S| \geq|A|-\varepsilon n$.

Lemma 14 (Lemma 5.6 in [9]). Let $G$ be of type $I(q)$. If $S \subset G$ is a sum-free set and $|S|>$ $\left(\frac{1}{3}+\frac{1}{3(q+1)}\right) n$, then $S \subset B$ for some largest sum-free set $B \subset G$.

Lemma 15 (Lemma 5.2 in [9]). Suppose that $G$ is abelian, $S \subset G$ a sum-free set, $r>0$ and $|S| \geq n / 3+r$. Then there is a subgroup $H$ of $G$ with $|H| \geq 3 r$, and a sum-free set $T \subset G / H$ such that $S \subseteq \pi^{-1}(T)$, where $\pi: G \rightarrow G / H$ is the canonical homomorphism. 
Further we will need the following result from [2] (see page 19).

Lemma 16. Let $G$ be a type $I(q)$ group of odd order and let $B \subset G$ be a largest sum-free set. If $x \in G \backslash B$, then

$$
\left|\left\{\{a, b\} \in\left(\begin{array}{c}
B \\
2
\end{array}\right): x=a+b\right\}\right| \geq \frac{n}{2 q}-1 .
$$

Even order groups. For even order groups we need further results. The first one refines and improves Lemma 14 for even order groups as follows.

Lemma 17. Let $G$ be an abelian group of even order and let $S \subset G$ be sum-free. If $|S|>\frac{3}{8} n$ and $S$ is not contained in a largest sum-free set of $G$, then there exist a subgroup $H$ of $G$ with $|H|=\frac{n}{5}$ and a largest sum-free set $T \subset G / H$ such that $S \subseteq \pi^{-1}(T)$, where $\pi: G \rightarrow G / H$ is the canonical homomorphism. Moreover, for all largest sum-free sets $B$ of $G$ we have $\left|\pi^{-1}(T) \cap B\right|=\frac{n}{5}$.

In particular, if $|S|>\frac{2}{5} n$, then $S$ is contained in a largest sum-free set of $G$.

Proof. Let $H$ be the subgroup of $G$ and $T$ be the sum-free set of $G / H$ obtained by the application of Lemma 15 with $r=\frac{n}{24}$. Then, $S \subset \pi^{-1}(T)$ and $|H|=\frac{n}{\ell}$ for some $\ell \in\{2, \ldots, 8\}$. If $\ell$ is even then $G / H$ has largest sum-free set of size $\ell / 2$. Hence, If $T$ is a largest sum-free set in $G / H$, then $S$ is contained in a largest sum-free set in $G$, a contradiction. If, on the other hand, $T$ is not a largest sum-free set in $G / H$, then trivially $\left|\pi^{-1}(T)\right| \leq \frac{n}{\ell}\left(\frac{\ell}{2}-1\right) \leq \frac{3}{8} n$, a contradiction to the size of $S$.

Suppose now that $\ell \in\{3,7\}$. Then $G / H$ is isomorphic to $\mathbb{Z}_{3}$ or to $\mathbb{Z}_{7}$. As the largest sum-free sets in $\mathbb{Z}_{3}$ and $\mathbb{Z}_{7}$ have sizes one and two, respectively, we have $\left|\pi^{-1}(T)\right| \leq \frac{1}{3} n<\frac{3}{8} n$, which is a contradiction to the size of $S$. Hence, $\ell=5$ which finishes the proof of the first part of the lemma.

Suppose now that $B$ is a largest sum-free set in $G$. Then $B^{0}=G \backslash B$ is an index two subgroup of $G$ due to Theorem 11], Since $G /\left(H \cap B^{0}\right) \simeq G / H \oplus G / B^{0} \simeq \mathbb{Z}_{5} \oplus \mathbb{Z}_{2}$, the set $B$ must correspond to $\mathbb{Z}_{5} \oplus\{1\}$ and $\pi^{-1}(T)$ corresponds to $\{1,4\} \oplus \mathbb{Z}_{2}$ or to $\{2,3\} \oplus \mathbb{Z}_{2}$. We conclude $\left|\pi^{-1}(T) \cap B\right|=\frac{n}{5}$.

Lemma 18. Let $G$ be an abelian group of even order, let $\mathcal{B}=\left(B_{1}, \ldots, B_{t}\right)$ be an ordered tuple of largest sum-free sets in $G$ and $x \in \mathcal{B}\left(\mathbf{0}_{t}\right)$ where $\mathbf{0}_{t}$ is the zero vector of length $t$. Then for every $\varepsilon \in\{0,1\}^{t}$ and every $b \in \mathcal{B}(\varepsilon)$, we have $x-b \in \mathcal{B}(\varepsilon)$.

Proof. Let $b^{\prime}=x-b$ and for a contradiction suppose that $b^{\prime} \in \mathcal{B}\left(\varepsilon^{\prime}\right)$ for an $\varepsilon^{\prime}=\left(\varepsilon_{1}^{\prime}, \ldots, \varepsilon_{t}^{\prime}\right) \neq$ $\varepsilon=\left(\varepsilon_{1}, \ldots, \varepsilon_{t}\right)$. Then there is an index $i$ such that $\varepsilon_{i}^{\prime} \neq \varepsilon_{i}$, i.e., we have that one of the two elements $b$ or $b^{\prime}$ is contained in $B_{i}^{0}$ but not both. However, as $x \in B_{i}^{0}$, this yields a contradiction to the fact that, due to Theorem 11, $B_{i}^{0}=G \backslash B_{i}$ is a subgroup of $G$. The lemma follows.

Given an even order group $G$ and a tuple of largest sum-free sets. The following lemma determines the size of the atoms and characterizes all largest sum-free sets contained in this tuple. It is a crucial part in our reduction of the original problem in arbitrary even order groups to a related one in $\mathbb{F}_{2}^{t}$ for some $t \leq r$. 
Lemma 19. Let $G$ be an abelian group of even order and let $\mathcal{B}=\left(B_{1}, \ldots, B_{t}\right), t \geq 2$, be an independent tuple of largest sum-free sets in $G$. Further, let $\mathbf{0}_{t}$ denote the zero vector of length $t$. Then the size of each atom of $\mathcal{B}$ is $n / 2^{t}$ and, $B \subseteq \bigcup_{i \in[t]} B_{i}$ is a largest sum-free set in $G$ if and only if there is a largest sum-free set $S$ of $\mathbb{F}_{2}^{t}$ such that

$$
B=\bigcup_{\varepsilon \in S} \mathcal{B}(\varepsilon)
$$

Proof. To show the lemma we will prove that the atoms $\mathcal{B}(\varepsilon), \varepsilon \in\{0,1\}^{t}$ are exactly the cosets of $\mathcal{B}\left(\mathbf{0}_{t}\right)$, i.e., $G / \mathcal{B}\left(\mathbf{0}_{t}\right)=\left\{\mathcal{B}(\varepsilon): \varepsilon \in\{0,1\}^{t}\right\}$, that $\mathcal{B}\left(\mathbf{0}_{t}\right)$ has size $n / 2^{t}$ and that the map $\pi: G / \mathcal{B}\left(\mathbf{0}_{t}\right) \rightarrow \mathbb{F}_{2}^{t}$ defined via $\pi(\mathcal{B}(\varepsilon))=\varepsilon$ is a group isomorphism.

To see that this implies the conclusion of the lemma note first that a (largest) sum-free set $S \subset \mathbb{F}_{2}^{t}$ of size $2^{t-1}$ maps to the sum-free set $\pi^{-1}(S) \subset G / \mathcal{B}\left(\mathbf{0}_{t}\right)$ of the same size. The union $B$ of the cosets of $\pi^{-1}(S)$ is then a (largest) sum-free set in $G$ of size $n / 2$. As $\pi^{-1}(S)$ cannot contain $\mathbf{0}_{t}$ we have $B \subseteq \bigcup_{i \in[t]} B_{i}$. On the other hand, if $B \subseteq \bigcup_{i \in[t]} B_{i}$ is a largest sum-free set in $G$, then $B^{0}=G \backslash B$ is a subgroup of $G$ due to Theorem [11, which properly contains $\mathcal{B}\left(\mathbf{0}_{t}\right)$ since $t \geq 2$. By the third isomorphism theorem $B^{0} / \mathcal{B}\left(\mathbf{0}_{t}\right)$ is then a non-trivial subgroup of $G / \mathcal{B}\left(\mathbf{0}_{t}\right)$ and the union of the cosets in $B^{\prime}=\left(G / \mathcal{B}\left(\mathbf{0}_{t}\right)\right) \backslash\left(B^{0} / \mathcal{B}\left(\mathbf{0}_{t}\right)\right)$ is $B$. As $B$ is largest sum-free and each coset has size $n / 2^{t}$ we have that $B^{\prime}$ is sum-free in $G / \mathcal{B}\left(\mathbf{0}_{t}\right)$ and has size $2^{t-1}$. Hence, $B^{\prime}$ corresponds to a largest sum-free set in $\mathbb{F}_{2}^{t}$ via $\pi$.

We proceed with the proof of the lemma and first note that each non-empty atom $\mathcal{B}(\varepsilon)$, $\varepsilon \in\{0,1\}^{t}$, is a subset of a coset of $\mathcal{B}\left(\mathbf{0}_{t}\right)$ in $G$, i.e., that $a-a^{\prime} \in B_{i}^{0}$ holds for all $a, a^{\prime} \in \mathcal{B}(\varepsilon)$ and all $i \in[t]$. Indeed, recall that $B_{i}^{0}=G \backslash B_{i}$ is a subgroup of $G$. Hence, if $\varepsilon_{i}=0$ then $a, a^{\prime} \in B_{i}^{0}$ and therefore $a-a^{\prime} \in B_{i}^{0}$. On the other hand, if $\varepsilon_{i}=1$, then $a$ and $a^{\prime}$ are in the largest sum-free set $B_{i}$. Thus, $a+a^{\prime}$ and $2 a^{\prime}$ are in its complement, the subgroup $B_{i}^{0}$. Therefore $-2 a^{\prime}$ is also in $B_{i}^{0}$ and we have $a-a^{\prime}=a+a^{\prime}-2 a^{\prime} \in B_{i}^{0}$. This shows that $\mathcal{B}(\varepsilon)$ is a subset of a coset of $\mathcal{B}\left(\mathbf{0}_{t}\right)$.

Further, it is easily seen that two non-empty atoms $\mathcal{B}(\varepsilon) \neq \mathcal{B}\left(\varepsilon^{\prime}\right)$ are contained in different cosets of $\mathcal{B}\left(\mathbf{0}_{t}\right)$. Indeed, as $\varepsilon \neq \varepsilon^{\prime}$ there is an index $i \in[t]$ such that, say, $\varepsilon_{i}=0$ and $\varepsilon_{i}^{\prime}=1$. Given $a \in \mathcal{B}(\varepsilon)$ and $a^{\prime} \in \mathcal{B}\left(\varepsilon^{\prime}\right)$, then $a-a^{\prime}$ cannot belong to $\mathcal{B}\left(\mathbf{0}_{t}\right)$ since $B_{i}^{0}$ is a subgroup. As the atoms form a partition of $G$ we conclude that they are exactly the cosets of $\mathcal{B}\left(\mathbf{0}_{t}\right)$.

To show $\left|\mathcal{B}\left(\mathbf{0}_{t}\right)\right|=n / 2^{t}$ we note that for all $k \in[t-1]$ the following holds

$$
\mathcal{B}\left(\mathbf{0}_{k}\right)=\left(\mathcal{B}\left(\mathbf{0}_{k}\right) \cap B_{k+1}^{0}\right) \cup\left(\mathcal{B}\left(\mathbf{0}_{k}\right) \cap B_{k+1}\right)=\mathcal{B}\left(\mathbf{0}_{k+1}\right) \cup \mathcal{B}(0, \ldots, 0,1) .
$$

Here $\mathcal{B}\left(\mathbf{0}_{k+1}\right)$ is a subgroup of $\mathcal{B}\left(\mathbf{0}_{k}\right)$ and $\mathcal{B}(0, \ldots, 0,1)$ is a non-empty set due to the lemma's assumption that $B_{k+1}$ is not contained in the union of the remaining $B_{i}$ 's. The argument from above yields therefore that $\mathcal{B}(0, \ldots, 0,1)$ is a coset of $\mathcal{B}\left(\mathbf{0}_{k+1}\right)$ in the group $\mathcal{B}\left(\mathbf{0}_{k}\right)$ which implies that $\left|\mathcal{B}\left(\mathbf{0}_{k+1}\right)\right|=|\mathcal{B}(0, \ldots, 0,1)|=\left|\mathcal{B}\left(\mathbf{0}_{k}\right)\right| / 2$. With $\left|\mathcal{B}\left(\mathbf{0}_{1}\right)\right|=\left|B_{1}^{0}\right|=n / 2$, due to Theorem 11, we obtain that $\left|\mathcal{B}\left(\mathbf{0}_{t}\right)\right|=n / 2^{t}$.

It is left to verify that the bijective map from $G / \mathcal{B}\left(\mathbf{0}_{t}\right)$ to $\mathbb{F}_{2}^{t}$ defined by $\mathcal{B}(\varepsilon) \mapsto \varepsilon$ is a group homomorphism, i.e., that for all $\varepsilon, \varepsilon^{\prime} \in\{0,1\}^{t}$ we have $\mathcal{B}(\varepsilon)+\mathcal{B}\left(\varepsilon^{\prime}\right)=\mathcal{B}\left(\varepsilon+\varepsilon^{\prime}\right)$ where the first addition is in $G / \mathcal{B}\left(\mathbf{0}_{t}\right)$ and the second is in $\mathbb{F}_{2}^{t}$. This can be verified component-wise, noting that 
for a fixed $i \in[t]$ we have $B_{i}^{0}+B_{i}^{0}=B_{i}^{0}$, because $B_{i}^{0}$ is a subgroup, and that $B_{i}^{1}+B_{i}^{1}=B_{i}^{0}$, due to Theorem [1]. Further, as $B_{i}^{1}$ is the only coset of $B_{i}^{0}$ in $G$ we have $B_{i}^{1}+B_{i}^{0}=B_{i}^{1}$. The lemma follows.

The characterization of the largest sum-free sets in the additive group $\mathbb{F}_{2}^{t}$ is immediate from Theorem 11 and the fact that $\mathbb{F}_{2}^{t}$ has exactly $2^{t}-1$ subgroups of index two, given by $\left\{\left(a_{1}, \ldots, a_{t}\right) \in \mathbb{F}_{2}^{t}: \sum_{i \in I} a_{i} \equiv 0(\bmod 2)\right\}$ for non-empty $I \subseteq[t]$.

Lemma 20. A subset $S \subseteq \mathbb{F}_{2}^{t}$ is a largest sum-free set in $\mathbb{F}_{2}^{t}$ if and only if there exists a non-empty $I \subseteq[t]$ such that

$$
S=\left\{\left(a_{1}, \ldots, a_{t}\right) \in \mathbb{F}_{2}^{t}: \sum_{i \in I} a_{i} \equiv 1 \quad(\bmod 2)\right\} .
$$

Given an independent tuple $\mathcal{B}=\left(B_{1}, \ldots, B_{t}\right), t \geq 2$, of largest sum-free sets in $G$. As a consequence of Lemmas 19 and 20 there is a correspondence between largest sum-free sets of $B \subset \cup_{i \in[t]} B_{i}$ of $G$ and non-empty subsets $I_{B} \subset[t]$. The one which assigns $B_{i}$ to $\{i\}$ for each $i \in[t]$ is called the canonical correspondence. It is easily seen that this indeed yields a correspondence, e.g., by induction on $t$. We summarize the properties of such a correspondence.

Corollary 21. Let $G$ be an abelian group of even order and let $\mathcal{B}=\left(B_{1}, \ldots, B_{t}\right), t \geq 2$, be an independent tuple of largest sum-free sets in $G$. Consider the canonical correspondence between largest sum-free sets $B \subset \cup_{i \in[t]} B_{i}$ of $G$ and non-empty subsets $I_{B} \subset[t]$. Then the size of each atom of $\mathcal{B}$ is $n / 2^{t}$ and an atom $\mathcal{B}(\varepsilon)$ is contained in $B$ if and only if $\varepsilon=\left(\varepsilon_{1}, \ldots, \varepsilon_{t}\right)$ evaluates odd on $I_{B}$, i.e. $\sum_{i \in I_{B}} \varepsilon_{i}$ is odd.

We finish the section with the proof of Proposition 8 showing that the following holds for sum-free sets $B_{1}, B_{2}, B_{3}$ in even order groups which satisfy $\left|\left\{B_{1}, B_{2}, B_{3}\right\}\right| \geq 2$ :

$$
\kappa_{4}\left(B_{1} \cup B_{2}\right) \geq(3 \sqrt{2})^{n / 2} \quad \text { and } \quad \kappa_{5}\left(B_{1} \cup B_{2} \cup B_{3}\right) \geq 6^{n / 2} .
$$

Proof of Proposition 8. Given $G$ of even order with an independent tuple $\mathcal{B}=\left(B_{1}, B_{2}\right)$ of two largest sum-free sets. Consider the canonical correspondence (see Corollary 21), relating $B_{i}$, $i \in[2]$, to the set $\{i\}$. Each atom of $\mathcal{B}$ has size $n / 4$ and $\mathcal{B}(1,0), \mathcal{B}(1,1)$ are those of $B_{1}$ whereas those of $B_{2}$ are $\mathcal{B}(0,1), \mathcal{B}(1,1)$. Further, there is a largest sum-free set $B_{3}$ corresponding to $\{1,2\}$ with the atoms $\mathcal{B}(1,0)$ and $\mathcal{B}(0,1)$, i.e, $B_{3}=B_{1} \triangle B_{2}$.

For the first part of the proposition consider all $\varphi: B_{1} \cup B_{2} \rightarrow[4]$ such that $\varphi^{-1}(1) \subseteq B_{1}$, $\varphi^{-1}(2) \subseteq B_{2}$ and $\varphi^{-1}(3), \varphi^{-1}(4) \subseteq B_{3}$. These colorings are clearly sum-free and there are $3^{n / 2} 2^{n / 4}$ of them, as the elements in $\mathcal{B}(1,1)$ can be colored with the colors 1 and 2 , the elements in $\mathcal{B}(1,0)$ with colors 1,3 and 4 and the elements in $\mathcal{B}(0,1)$ with colors 2,3 and 4.

For the second part first consider the case that $B_{1}$ and $B_{2}$ are two sum-free sets and $B_{3}=$ $B_{1} \triangle B_{2}$ as above. Consider the colorings $\varphi: B_{1} \cup B_{2} \rightarrow[5]$ such that $\varphi^{-1}(1), \varphi^{-1}(2) \subseteq B_{1}$, $\varphi^{-1}(3), \varphi^{-1}(4) \subseteq B_{2}$ and $\varphi^{-1}(5) \subseteq B_{3}$. This gives rise to $3^{n / 2} 4^{n / 4}$ sum-free 5 -colorings. 
Finally, given the independent triple $\mathcal{B}=\left(B_{1}, B_{2}, B_{3}\right)$ with atoms of size $n / 8$ each, because of Corollary 21, Consider the canonical correspondence relating $B_{i}, i \in[3]$, to the set $\{i\}$. Then there are four further largest sum-free sets contained in $B_{1} \cup B_{2} \cup B_{3}$. One of these is $B_{4}=B_{1} \triangle B_{2}$ corresponding to $\{1,2\}$ and consisting of the atoms $\mathcal{B}(1,0,0), \mathcal{B}(1,0,1), \mathcal{B}(0,1,0), \mathcal{B}(0,1,1)$. An another one is $B_{5}=\left(B_{1} \triangle B_{2} \triangle B_{3}\right) \cup\left(B_{1} \cap B_{2} \cap B_{3}\right)$ corresponding to $\{1,2,3\}$ with the atoms $\mathcal{B}(1,0,0), \mathcal{B}(0,1,0), \mathcal{B}(0,0,1), \mathcal{B}(1,1,1)$. Consider all colorings $\varphi: B_{1} \cup B_{2} \cup B_{3} \rightarrow[5]$ such that $\varphi^{-1}(i) \subset B_{i}, i \in[5]$. This gives rise to $3^{4 \cdot \frac{n}{8}} 2^{2 \cdot \frac{n}{8}} 4^{\frac{n}{8}}$ sum-free 5 -colorings.

\section{Proofs of stability: Theorem 7, 9, 10,}

With the group theoretic results from previous section we are now ready to give the proofs of Theorem 7. Theorem 9 and Theorem 10.

Proof of Theorem 7 . We give the proof for $r=3$ only. The proof for $r=2$ follows the same line. For given $\varepsilon>0$ let $\delta=\varepsilon / 200$ and let $|G|=n$ be sufficiently large.

Let $A \subset G$ with $\kappa_{3}(A) \geq 3^{\mu(G)-\delta n}$ and let $\mathcal{F}=\mathcal{F}_{A}$ be a container family as in Theorem 5 . According to (3) of this theorem each $F_{i}$ contains at most $n^{2}(\log n)^{-1 / 9}$ Schur triples and Lemma 12 implies for large enough $n$ that $\left|F_{i}\right| \leq \mu(G)+3 n(\log n)^{-1 / 27}$ for each $F_{i} \in \mathcal{F}$. Let $\left(F_{1}, F_{2}, F_{3}\right)$ be a triple maximizing $\left|\Phi\left(F_{1}^{\prime}, F_{2}^{\prime}, F_{3}^{\prime}\right)\right|$ over all $\left(F_{1}^{\prime}, F_{2}^{\prime}, F_{3}^{\prime}\right) \in \mathcal{F}^{3}$. Together with (1) of Observation 6 we infer $3^{\mu(G)-\delta n} \leq \kappa_{3}(A) \leq|\mathcal{F}|^{3} \cdot\left|\Phi\left(F_{1}, F_{2}, F_{3}\right)\right|$. Further, (11) of Theorem 5 states that $\log _{2}|\mathcal{F}| \leq n(\log n)^{-1 / 18}$ which implies $\log _{3}\left|\Phi\left(F_{1}, F_{2}, F_{3}\right)\right| \geq \mu(G)-2 \delta n$ for large enough $n$.

Let $n_{i}, i=1,2,3$, be the number of elements contained in exactly $i$ members from $\left(F_{1}, F_{2}, F_{3}\right)$. Then $n_{1}+2 n_{2}+3 n_{3}=\left|F_{1}\right|+\left|F_{2}\right|+\left|F_{3}\right| \leq 3 \mu(G)+3 \delta n$, hence, $n_{2} \leq \frac{3}{2}\left(\mu(G)+\delta n-n_{3}\right)$. We conclude that $n_{3} \geq \mu(G)-59 \delta n$ must hold otherwise the first part of (2) of Observation 6 together with $\frac{3}{2} \log _{3} 2<\frac{19}{20}<1$ would yield the following contradiction

$$
\begin{aligned}
\mu(G)-2 \delta n \leq \log _{3}\left|\Phi\left(F_{1}, F_{2}, F_{3}\right)\right| & \leq n_{3}+\left(\mu(G)+\delta n-n_{3}\right) \frac{3}{2} \log _{3} 2 \\
& <\mu(G)-59 \delta n+60 \delta n \cdot \frac{3}{2} \log _{3} 2<\mu(G)-2 \delta n .
\end{aligned}
$$

Let $F=F_{1} \cap F_{2} \cap F_{3}$ and note that since $A=F_{1} \cup F_{2} \cup F_{3}$ and $\left|F_{i} \backslash F\right|=\left|F_{i}\right|-|F| \leq 60 \delta n$ for $i=1,2,3$, we have $|A \backslash F| \leq 180 \delta n$. To conclude the proof all is needed is to show that $|F \backslash B| \leq \delta n$ for some largest sum-free set $B$ since this then implies $|A \backslash B| \leq 181 \delta n<\varepsilon n$.

Note to this end that $|F|=n_{3} \geq \mu(G)-59 \delta n=\left(\frac{1}{3}+\frac{1}{3 q}-59 \delta\right) n>\frac{n}{3}+\delta n$ due to $\delta \leq$ $\frac{1}{200(q+1)}$. Lemma 13 then implies that for sufficiently large $n$ there is a sum-free set $S$ of size $|S| \geq|F|-\delta n \geq \mu(G)-60 \delta n>\left(\frac{1}{3}+\frac{1}{3(q+1)}\right) n$ that is contained in $F$. By Lemma 14 there must exist a largest sum-free set $B \subset G$ such that $S \subset B$ showing that $|F \backslash B| \leq \delta n$, as claimed.

The proofs of Theorem 9 and Theorem 10 will be more involved and we divide the argument into two parts reflected by two lemmas, Lemma 22 and Lemma 23. In the following we state the two lemmas which will immediately imply Theorem 9 and Theorem 10, The proofs of the lemmas will be shown subsequently. 
The first lemma states that $\left|\Phi\left(F_{1}, \ldots, F_{r}\right)\right|$ being large implies that each of the $F_{i}$ 's is essentially contained in a largest sum-free set in $G$.

Lemma 22. Let $G$ be an abelian group of sufficiently large even order $n$. Given $A \subset G$ and $\mathcal{F}=\mathcal{F}(A)$ as in Theorem 5 and suppose that $G, r$ and $\left(F_{1}, \ldots, F_{r}\right) \in \mathcal{F}^{r}$ are such that

- $G$ has a unique largest sum-free set and $\left|\Phi\left(F_{1}, \ldots, F_{r}\right)\right|>\left(\sqrt{r}-\frac{1}{1000}\right)^{n}$ or

- $r=4$ and $G$ has at least two largest sum-free sets and $\left|\Phi\left(F_{1}, \ldots, F_{r}\right)\right|>2.01^{n}$ or

- $r=5$ and $G$ has at least two largest sum-free sets and $\left|\Phi\left(F_{1}, \ldots, F_{r}\right)\right|>2.41^{n}$.

Then there is an $r$-tuple of largest sum-free sets $\left(B_{1}, \ldots, B_{r}\right)$ in $G$ such that

$$
\left|F_{i} \backslash B_{i}\right| \leq 3 n(\log n)^{-1 / 27} \quad \text { for all } i \in[r] .
$$

Note that Theorem 9 and Theorem 10 immediately follows in case $G$ has a unique largest sum-free set. For the case that $G$ has at least two largest sum-free sets we put forth the following lemma, which determines the intersection structure of the optimal configuration of the $B_{i}$ 's.

Lemma 23. Given an abelian group $G$ of even order.

If $\mathcal{B}=\left(B_{1}, \ldots, B_{4}\right)$ is tuple of largest sum-free sets such that $\left|\Phi\left(B_{1}, \ldots, B_{4}\right)\right|>2^{n}$, then there are two sets among $B_{1}, \ldots, B_{4}$ whose union contains all $B_{i}, i=1, \ldots, 4$, and $\mathcal{B}$ consists of one 2-atom and two 3-atoms. In particular, $|\Phi(\mathcal{B})|=(3 \sqrt{2})^{n / 2}$.

If $\mathcal{B}=\left(B_{1}, \ldots, B_{5}\right)$ is a tuple of largest sum-free sets with $\left|\Phi\left(B_{1}, \ldots, B_{5}\right)\right|>\left(3 \cdot 2^{7 / 2}\right)^{n / 4}$. Then either

- there are two sets among $B_{1}, \ldots, B_{5}$ whose union contains all $B_{i}, i=1, \ldots, 5$, and $\mathcal{B}$ consists of two 3-atoms and one 4-atom, or

- there are three sets among $B_{1}, \ldots, B_{5}$ whose union contains all $B_{i}, i=1, \ldots, 5$, and $\mathcal{B}$ consists of two 2-atoms, four 3-atoms and one 4-atom.

In both cases we have $|\Phi(\mathcal{B})|=6^{n / 2}$.

Theorem 9 and Theorem 10 are now easy consequences of the two lemmas.

Proof of Theorem 9 and Theorem [10. Given an abelian group $G$ of sufficiently large even order $n$ and let $\gamma=3(\log n)^{-1 / 27}$. Let $A \subset G, \kappa_{r}(A)$ and $\mathcal{F}=\mathcal{F}(A)$ be as stated in Theorem 9 or Theorem 10, respectively. In the case that $G$ has a unique largest sum-free set $B$ let $\left(F_{1}, \ldots, F_{r}\right)$ be the tuple among all $\left(F_{1}^{\prime}, \ldots, F_{r}^{\prime}\right) \in \mathcal{F}^{r}$ which maximizes $\left|\Phi\left(F_{1}^{\prime}, \ldots, F_{r}^{\prime}\right)\right|$. Owing to (10) of Observation 6 we have $\kappa_{r}(A)=\left|\Phi_{r}(A)\right| \leq|\mathcal{F}|^{r}\left|\Phi\left(F_{1}, \ldots, F_{r}\right)\right|$. As $|\mathcal{F}|^{r} \leq 2^{r n(\log n)^{-1 / 18}} \ll \kappa_{r}(A)$ we conclude for sufficiently large $n$ that $\left|\Phi\left(F_{1}, \ldots, F_{r}\right)\right|>|\mathcal{F}|^{-r}\left(r-\frac{1}{1000}\right)^{n / 2}>\left(\sqrt{r}-\frac{1}{1000}\right)^{n}$ for $r=4,5$.

On the other hand, if $G$ has at least two largest sum-free sets let $\left(F_{1}, \ldots, F_{r}\right)$ be a tuple given by the theorems, i.e.,

- for $r=4$ we have $\left|\Phi\left(F_{1}, \ldots, F_{4}\right)\right|>\left(3 \sqrt{2}-\frac{1}{25}\right)^{n / 2}>2.01^{n}>4^{4 \gamma n} \cdot 2^{n}$ and

- for $r=5$ we have $\left|\Phi\left(F_{1}, \ldots, F_{5}\right)\right|>5.9^{n / 2}>5^{5 \gamma n}\left(3 \cdot 2^{7 / 2}\right)^{n / 4}>2.41^{n}$. 
In particular, the presumptions of Lemma 22 are satisfied in all cases and we infer that there is a tuple $\mathcal{B}=\left(B_{1}, \ldots, B_{r}\right)$ of largest sum-free sets in $G$ such that $\left|F_{i} \backslash B_{i}\right| \leq \gamma n$ for all $i \in[r]$. As $A=F_{1} \cup \cdots \cup F_{r}$ we have $|A \backslash B| \leq r \gamma n$ and the theorems follow in the case $G$ has a unique largest sum-free set.

Suppose now that $G$ has at least two largest sum-free sets. We aim to apply Lemma 23 to $\mathcal{B}$ from above and therefore now verify its presumption. Define for all $i \in[r]$ the sets $F_{i}^{1}=F_{i} \cap B_{i}$ and $F_{i}^{0}=F_{i} \backslash B_{i}$. Then $\left|\Phi\left(F_{1}, \ldots, F_{r}\right)\right| \leq\left|\Phi\left(F_{1}^{0}, \ldots, F_{r}^{0}\right)\right| \cdot\left|\Phi\left(F_{1}^{1}, \ldots, F_{r}^{1}\right)\right|$ and $\left|\Phi\left(F_{1}^{0}, \ldots, F_{r}^{0}\right)\right| \leq$ $r^{r \gamma n}$. Further, any coloring $\varphi \in \Phi\left(F_{1}^{1}, \ldots, F_{r}^{1}\right)$ can be extended to a coloring $\varphi^{\prime} \in \Phi\left(B_{1}, \ldots, B_{r}\right)$ as $F_{i}^{1} \subset B_{i}$ and $B_{i}$ is sum-free. This implies $\left|\Phi\left(F_{1}^{1}, \ldots, F_{r}^{1}\right)\right| \leq\left|\Phi\left(B_{1}, \ldots, B_{r}\right)\right|$ and we infer $|\Phi(\mathcal{B})| \geq r^{-r \gamma n}\left|\Phi\left(F_{1}, \ldots, F_{r}\right)\right|$. Considering the bounds on $\left|\Phi\left(F_{1}, \ldots, F_{r}\right)\right|$ from above, this shows that $\mathcal{B}$ satisfies the presumptions of Lemma 23 and the theorems follow from the conclusions of this lemma for the case $G$ has at least two largest sum-free sets.

4.1. Proofs of Lemma 22 and Lemma 23. We first show the proof of Lemma 23. Note that Corollary 21 reduces the problem to a finite task which one could check exhaustively. For completeness we give a self-contained proof.

Proof of Lemma 23. Given the tuple $\mathcal{B}=\left(B_{1}, \ldots, B_{r}\right)$ with the properties stated in the lemma. Let $n_{i}=\sum_{\varepsilon: \sum \varepsilon_{j}=i}|\mathcal{B}(\varepsilon)|$ be the number of elements contained in $i$-atoms, i.e., those belonging to exactly $i$ sets from $\mathcal{B}$. Recall from (2) of Observation $[6$ that

$$
\log _{3}|\Phi(\mathcal{B})| \leq \sum_{i \in[r]} n_{i} \log _{3} i \quad \text { and } \quad \sum_{i \in[r]} i \cdot n_{i}=\sum_{i \in[r]}\left|B_{i}\right|=r \mu(G)=\frac{r n}{2} .
$$

As $\frac{i}{3} \geq \log _{3} i$ for all integer $i \geq 1$ we derive for $\eta_{4}=\log _{3} 2$ and $\eta_{5}=13 / 16$ that

$$
\eta_{r} n<\log _{3}|\Phi(\mathcal{B})| \leq \sum_{i \in[r]} n_{i} \log _{3} i \leq \frac{1}{3} \sum_{i \in[r]} i \cdot n_{i}-\frac{1}{3} n_{1} \leq \frac{1}{3}\left(\frac{r n}{2}-n_{1}\right) .
$$

Let $t \in[r]$ be the largest number such that there is an independent $t$-tuple of elements in $\mathcal{B}$, which we denote by $\mathcal{A}=\left(A_{1}, \ldots, A_{t}\right)$. Recall the correspondence of the structure of $\mathcal{A}$ to that of $\mathbb{F}_{2}^{t}$ as described in Corollary 21. According to this each largest sum-free set $B$ contained in $\cup A_{i}$ corresponds to a largest sum-free set in $\mathbb{F}_{2}^{t}$ and is associated to a set $I=I(B) \subset[t]$. The atoms of $\mathcal{A}$ then correspond to elements $\varepsilon \in \mathbb{F}_{2}^{t}$ and an atom $\mathcal{A}(\varepsilon)$ is contained in $B$ if and only if $\varepsilon=\left(\varepsilon_{1}, \ldots, \varepsilon_{t}\right)$ evaluates odd on $I=I(B)$, i.e. $\sum_{i \in I} \varepsilon_{i}$ is odd. Throughout the proof we will consider the canonical correspondence assigning $A_{i}, i \in[t]$, to the set $I=\{i\}$, so that, the 1-atoms correspond to those $t$ elements $\varepsilon \in \mathbb{F}_{2}^{t}$ with exactly one 1-entry.

Using this correspondence we first show that $1<t \leq r-2$ and $n_{1}=0$. The bound $t>1$ is trivial, since otherwise we would have $|\Phi(\mathcal{B})|=r^{\frac{n}{2}} \leq 3^{\eta_{r} n}$ which contradicts (4). Suppose that $t=r$. Then the atoms have size $\frac{n}{2^{r}}$ due to Corollary 21 and the number of 1-atoms is $r$. Hence, $n_{1}=r \frac{n}{2^{r}}$ which yields the contradiction $\log _{3}|\Phi(\mathcal{B})| \leq \eta_{r} n$. On the other hand, if $t \leq r-1$ and $n_{1}>0$ then $n_{1}$ has the size of at least one atom, i.e., $n_{1} \geq n / 2^{r-1}$ which again yield a violation of $\log _{3}|\Phi(\mathcal{B})|>\eta_{r} n$. Having $t=r-1$, say $A_{1}=B_{1}, \ldots, A_{r-1}=B_{r-1}$, and $n_{1}=0$, however, 
means that the remaining largest sum-free set $B \in\left\{B_{1}, \ldots, B_{r}\right\} \backslash\left\{A_{1}, \ldots, A_{r-1}\right\}$ must cover all 1 -atoms of $\mathcal{A}$. Since $A_{i}$ corresponds to $\{i\}$ the set $B$ must correspond to $I=[t]$. This determines the atom structure of $\mathcal{B}$ completely and it is readily checked that $\mathcal{B}$ then consists of six 2 -atoms and one 4 -atom in case $r=4$ and of ten 2-atoms and five 4-atoms in case $r=5$. With (3) this yields a contradiction to the lower bound $\log _{3}|\Phi(\mathcal{B})|>\eta_{r} n$.

Hence, let $1<t \leq r-2$ and $n_{1}=0$ in both cases $r=4$ and $r=5$. For $t=2$ and $r=4$ let $a_{i}$, $i=2,3,4$, denote the number of $i$-atoms of $\mathcal{B}=\left(B_{1}, \ldots, B_{r}\right)$. Similarly define $b_{i}, i=2, \ldots, 5$ for $t=2, r=5$ and $c_{i}, i=2, \ldots, 5$, for $t=3$ and $r=5$. Due to Corollary 21 we know that in the case $t=2\left(t=3\right.$, respectively) the set $\cup_{i \in[r]} B_{i}$ is the union of three (seven, respectively) atoms, each of size $n / 2^{t}$. This and the second part of (3) implies that

$$
\sum_{i=2}^{4} a_{i}=\sum_{i=2}^{5} b_{i}=3, \quad \sum_{i=2}^{5} c_{i}=7, \quad \sum_{i=2}^{4} i a_{i}=8, \quad \sum_{i=2}^{4} i b_{i}=10, \quad \sum_{i=2}^{5} i c_{i}=20 .
$$

Solving the equations yields that $\left(a_{2}, a_{3}, a_{4}\right) \in\{(2,0,1),(1,2,0)\}$. If $\left(a_{2}, a_{3}, a_{4}\right)=(2,0,1)$ then the first part of (3) yields $|\Phi(\mathcal{B})| \leq(4 \cdot 4)^{n / 4}=2^{n}$ which violates the lower bound on $|\Phi(\mathcal{B})|$. For $\left(a_{2}, a_{3}, a_{4}\right)=(1,2,0)$ we have $|\Phi(\mathcal{B})| \leq(2 \cdot 9)^{n / 4}$. Further, by considering largest sumfree sets in $\mathbb{F}_{2}^{2}$ corresponding to $I_{1}=\{1\}, I_{2}=\{2\}, I_{3}=I_{4}=\{1,2\}$, one obtains via the canonical correspondence a tuple $\left(B_{1}, \ldots, B_{4}\right)$ which consists of one 2-atoms and two 3-atoms, hence realizing the optimum $\left(a_{2}, a_{3}, a_{4}\right)=(1,2,0)$. The first part of the lemma follows.

Turning to the second part of the lemma we first solve (5) for $\left(b_{2}, \ldots, b_{5}\right)$ and obtain its solution set $\{(1,1,0,1),(1,0,2,0),(0,2,1,0)\}$. It is readily checked that $|\Phi(\mathcal{B})| \leq 6^{n / 2}$ where the maximum is achieved only for $\left(b_{2}, \ldots, b_{4}\right)=(0,2,1,0)$. The second largest value is attained by $(1,0,2,0)$ which would yield $|\Phi(\mathcal{B})| \leq(2 \cdot 16)^{n / 8}$, a contradiction to the lower bound. Further, by considering largest sum-free sets in $\mathbb{F}_{2}^{2}$ corresponding to $I_{1}=I_{2}=\{1\}, I_{3}=I_{4}=\{2\}$ and $I_{5}=\{1,2\}$ we see that the optimum $\left(b_{2}, \ldots, b_{4}\right)=(0,2,1,0)$ can indeed be realized.

Finally, we solve (5) for $\left(c_{2}, \ldots, c_{5}\right)$ and obtain its solution set $\{(1,6,0,0),(2,4,1,0),(3,2,2,0)$, $(3,3,0,1),(4,0,3,0),(4,1,1,1),(5,0,0,2)\}$. However, the maximum is attained by $(1,6,0,0)$, which cannot be realized as atom structure. To see this, suppose that $\mathcal{B}=\left(B_{1}, \cdots, B_{5}\right)$ has this atom structure. Assume that $\mathcal{B}^{\prime}=\left(B_{1}, B_{2}, B_{3}\right)$ is independent and corresponds to $I_{i}=\{i\}$. Note that $\mathcal{B}^{\prime}(1,1,1)$ is a 3 -atom, hence $B_{4}$ and $B_{5}$ cannot contain $\mathcal{B}^{\prime}(1,1,1)$ since $\mathcal{B}(1,1,1)$ would be a 4 -atom or a 5 -atom otherwise. However, largest sum-free sets of $\mathbb{F}_{2}^{3}$ which do not contain $(1,1,1)$ correspond to two elements sets $I \subset[3]$. In particular, they consist of two 1-atoms and two 2 -atoms of $\mathcal{B}^{\prime}$. As there are only three 2 -atoms in $\mathcal{B}^{\prime}$ we conclude that $\mathcal{B}$ would contain a 4-atom.

Within the solutions for $\left(c_{2}, \ldots, c_{5}\right)$, the third largest value is achieved by $(3,2,2,0)$ giving $|\Phi(\mathcal{B})| \leq(8 \cdot 9 \cdot 16)^{n / 8}$, a contradiction to the lower bound. The second largest is obtained by $(2,4,1,0)$ giving $|\Phi(\mathcal{B})| \leq(4 \cdot 81 \cdot 4)^{n / 8}=6^{n / 2}$. Moreover, the five largest sum-free sets corresponding to $I_{i}=\{i\}, i \in[3], I_{4}=\{1,2\}$ and $I_{5}=\{1,3\}$ indeed consists of two 2-atoms, four 3 -atoms and one 4 -atom showing that $(2,4,1,0)$ is indeed realizable. The lemma follows. 
Proof of Lemma 22. Let $\gamma=3(\log n)^{-1 / 27}$. Given an even order abelian group $G$ of sufficiently large order $n$ together with a container family $\mathcal{F}$ as in Theorem 5 and let $\left(F_{1}, \ldots, F_{r}\right)$ be a tuple as in the lemma. A set $F$ is called good if there is a largest sum-free set $B$ in $G$ such that $|F \backslash B| \leq \gamma n$. Hence, to establish the lemma we need to show that all $F_{i}, i=1, \ldots, r$, are good.

Note to this end that $\left|F_{i}\right|>\frac{2}{5} n+\gamma n$ readily implies that $F_{i}$ is good. Indeed, by property (3) of Theorem [5 we know that $F_{i}$ contains at most $n^{2}(\log n)^{-1 / 9}$ Schur triples. Lemma 13 then implies that one can obtain a sum-free set $S_{i} \subset F_{i}$ by removing at most $\gamma n$ elements from $F_{i}$. As $\left|S_{i}\right|>\frac{2}{5} n$ we conclude by the last part of Lemma 17 that there is a largest sum-free set $B_{i}$ such that $S_{i} \subset B_{i}$, showing that $F_{i}$ is good.

For any given tuple $\left(F_{1}, \ldots, F_{r}\right) \in \mathcal{F}^{r}$ of containers let $n_{i}$ denote the number of elements contained in exactly $i$ sets from $\left\{F_{1}, \ldots, F_{r}\right\}$. Recall from (2) that

$$
\left|\Phi\left(F_{1}, \ldots, F_{r}\right)\right| \leq \prod_{i \in[r]} i^{n_{i}} \quad \text { and } \quad \sum_{i \in[r]} i \cdot n_{i}=\sum_{i \in[r]}\left|F_{i}\right|
$$

holds. Using $\frac{i}{3} \geq \log _{3} i$ for all integer $i \geq 1$ we derive

$$
\log _{3}\left|\Phi\left(F_{1}, \ldots, F_{r}\right)\right| \leq \frac{1}{3} \sum_{i \in[r]}\left|F_{i}\right| .
$$

Further, by (3) of Theorem 5 and Lemma 12 we have $\left|F_{i}\right| \leq \frac{n}{2}+2^{20} n(\log n)^{-1 / 45}$ for all $i \in[r]$.

We first consider the case that $G$ has at least two largest sum-free sets for which the lemma easily follows. Indeed, suppose that there is an $i \in[r]$ such that $\left|F_{i}\right| \leq \frac{2}{5} n+\gamma n$. Then we have

$$
\sum_{i \in[r]}\left|F_{i}\right| \leq(r-1)\left(\frac{n}{2}+2^{20} \frac{n}{(\log n)^{\frac{1}{45}}}\right)+\frac{2}{5} n+\gamma n,
$$

which, with (7) and sufficiently large $n$, yields $\left|\Phi\left(F_{1}, \ldots, F_{r}\right)\right|<2.01^{n}$ in the case $r=4$ and $\left|\Phi\left(F_{1}, \ldots, F_{r}\right)\right|<2.41^{n}$ in the case $r=5$. This, however, yields a contradiction to the fact that $\left(F_{1}, \ldots, F_{r}\right)$ is substantial and we conclude that $\left|F_{i}\right|>\frac{2}{5} n+\gamma n$ for all $i \in[r]$ implying that $F_{i}$ is good, as claimed 2

Next we prove the lemma in case the group has a unique largest sum-free set which is somewhat more complicated as the lower bound is significantly smaller in this case. Let $G$ be a group of even order with the unique largest sum-free set $B$ and let $\left(F_{1}, \ldots, F_{r}\right)$ be a substantial tuple, i.e., $\left|\Phi\left(F_{1}, \ldots, F_{r}\right)\right| \geq(\sqrt{r}-0.01)^{n}$. Note first that if $F_{1}, \ldots, F_{r-1}$ of the tuple $\left(F_{1}, \ldots, F_{r}\right)$ are good, then with $F_{i}^{1}=F_{i} \cap B$ we have

$$
\left(r^{\frac{1}{2}}-0.01\right)^{n} \leq\left|\Phi\left(F_{1}, \ldots, F_{r}\right)\right| \leq r^{r \gamma n}\left|\Phi\left(F_{1}^{1}, \ldots, F_{r-1}^{1}, F_{r}\right)\right| \leq r^{r \gamma n} r^{\left|F_{r} \cap B\right|}(r-1)^{\left|B \backslash F_{r}\right|} .
$$

This implies that $F_{r}$ must have size larger than $\frac{2}{5} n+\gamma n$ for sufficiently large $n$ and hence being good as well.

\footnotetext{
${ }^{2}$ We note that the same argument also works for $r=6$ in conjunction with the lower bound $\kappa_{r, G} \geq \kappa_{6}\left(B_{1} \cup\right.$ $\left.B_{2} \cup B_{3}\right) \geq 2^{3 n / 4} 3^{n / 2} \geq 2.9129^{n}$ obtained by an independent triple $\left(B_{1}, B_{2}, B_{3}\right)$ of largest sum-free sets. Assuming that a there is an $i \in[6]$ with $\left|F_{i}\right| \leq \frac{2}{5} n+\gamma n$ would imply $\left|\Phi\left(F_{1}, \ldots, F_{r}\right)\right|<2.91^{n}$.
} 
Further, by the same argument as above, i.e., using $\left|F_{i}\right| \leq \frac{n}{2}+2^{20} n(\log n)^{-1 / 45}$ for all $i \in[r]$ together with property (7) we derive the following properties.

\section{Claim 23.1.}

(1) Let $k_{4}=3$ and $k_{5}=2$ then at least $k_{r}$ sets from $\left(F_{1}, \ldots, F_{r}\right)$ have size larger than $\frac{2}{5} n+\gamma n$, implying that they are good,

(2) at least four sets from $\left(F_{1}, \ldots, F_{5}\right)$ have size larger than $\frac{n}{3}+\gamma n$, and

(3) if three sets from $\left(F_{1}, \ldots, F_{5}\right)$ have size at most $\frac{2}{5} n+\gamma n$ then all five sets have size larger than $\frac{3}{8} n+\gamma n$.

In particular, as $k_{4}=3$ the lemma follows in the case $r=4$ and it is left to prove that for $r=5$ there are four sets out of $\left(F_{1}, \ldots, F_{5}\right)$ which have size larger than $\frac{2}{5} n+\gamma n$.

For a given tuple $\left(F_{1}, \ldots, F_{5}\right)$ let $F_{i}^{1}=F_{i} \cap B$ and $F_{i}^{0}=F_{i} \backslash B$ and let $m_{i}$ be the number of elements contained in exactly $i$ sets from $\left(F_{1}^{1}, \ldots, F_{5}^{1}\right)$. Then

(9) $\left|\Phi\left(F_{1}, \ldots, F_{5}\right)\right| \leq\left|\Phi\left(F_{1}^{0}, \ldots, F_{5}^{0}\right)\right| \cdot\left|\Phi\left(F_{1}^{1}, \ldots, F_{5}^{1}\right)\right| \quad$ and $\quad m_{1}+\cdots+m_{5}=|B|=\frac{n}{2}$.

Note also that $\left|\Phi\left(F_{1}^{1}, \ldots, F_{5}^{1}\right)\right| \leq\left|\Phi\left(B, \ldots, B, F_{i}^{1}, \ldots, F_{5}^{1}\right)\right|, i \in[5]$, since $F_{j}^{1} \subset B$, for all $j \in[5]$.

By (11) of Claim 23.1 we may assume that $F_{1}$ and $F_{2}$ are good, and for a contradiction suppose that $\left|F_{i}\right| \leq \frac{2}{5} n+\gamma n, i=3,4,5$. Then due to (3) of Claim 23.1 each of these $F_{i}$ 's has size larger than $\frac{3}{8} n+\gamma n$. Property (3) of Theorem 5 and Lemma 13 implies that for each such $i$ there is a sum-free set $S_{i} \subseteq F_{i}$ such that $\left|F_{i} \backslash S_{i}\right| \leq \gamma n$. By Lemma 17 there is a sum-free set $L_{i}$ containing $S_{i}$ such that $\left|L_{i} \cap B\right|=\left|L_{i} \backslash B\right|=\frac{n}{5}$. Therefore $\left|F_{i}^{0}\right|,\left|F_{i}^{1}\right| \leq \frac{n}{5}+\gamma n$ for $i \in\{3,4,5\}$ and (7) yields $\left|\Phi\left(F_{1}^{0}, \ldots, F_{r}^{0}\right)\right| \leq 3^{\frac{n}{5}+2 \gamma n}$.

Consider now the tuple $\left(B, B, F_{3}^{1}, F_{4}^{1}, F_{5}^{1}\right)$ with $f=\left|F_{3}^{1}\right|+\left|F_{4}^{1}\right|+\left|F_{5}^{1}\right| \leq \frac{3}{5} n+3 \gamma n$ and let $m_{i}$ be defined as above. Then $m_{3}+2 m_{4}+3 m_{5}=f$ and together with the second part of (9) this implies $m_{4}+2 m_{5}-m_{2}+\frac{n}{2}-f=0$. Using the first part of (9) and $\sqrt{\frac{20}{27}}<\frac{8}{9}$ we obtain for $G$ of sufficiently large order

$$
\begin{aligned}
\left|\Phi\left(F_{1}, \ldots, F_{5}\right)\right| & \leq\left|\Phi\left(F_{1}^{0}, \ldots, F_{5}^{0}\right)\right|\left|\Phi\left(B, B, F_{3}^{1}, F_{4}^{1}, F_{5}^{1}\right)\right| \\
& \leq 3^{\frac{n}{5}+2 \gamma n} 2^{m_{2}} 3^{m_{3}} 4^{m_{4}} 5^{m_{5}} \\
& =3^{\frac{n}{5}+2 \gamma n} 2^{m_{4}+2 m_{5}+\frac{n}{2}-f} 3^{f-2 m_{4}-3 m_{5}} 4^{m_{4}} 5^{m_{5}} \\
& \leq 3^{\frac{n}{5}+2 \gamma n} 2^{\frac{n}{2}-f} 3^{f}\left(\frac{8}{9}\right)^{m_{4}}\left(\frac{20}{27}\right)^{m_{5}} \\
& \leq 3^{\frac{n}{5}+2 \gamma n} 2^{\frac{n}{2}-f} 3^{f}\left(\frac{8}{9}\right)^{f-\frac{n}{2}} \\
& \leq 3^{\frac{n}{5}+2 \gamma n}\left(\frac{4}{3}\right)^{f}\left(\frac{3}{2}\right)^{n}<2.23^{n} .
\end{aligned}
$$

This contradicts the lower bound on $\left|\Phi\left(F_{1}, \ldots, F_{5}\right)\right|$ and we conclude that one set among $F_{3}, F_{4}, F_{5}$, say $F_{3}$, has size larger than $\frac{2}{5} n+\gamma n$ and is therefore good. 
Finally we show that $F_{4}$ or $F_{5}$ is good, assuming that $F_{1}, F_{2}, F_{3}$ are. Let $f_{0}=\left|F_{4}^{0} \cap F_{5}^{0}\right|$ and note that $\left|\Phi\left(F_{1}^{0}, \ldots, F_{5}^{0}\right)\right| \leq 5^{4 \gamma n} 2^{f_{0}}$ due to the goodness of $F_{1}, F_{2}$ and $F_{3}$. Further, consider $m_{1}, \ldots, m_{5}$ for the tuple $\left(B, B, B, F_{4}^{1}, F_{5}^{1}\right)$. Then with $f=\left|F_{4}\right|+\left|F_{5}\right|$ we have $m_{1}=m_{2}=0$ and

$$
m_{3}+m_{4}+m_{5}=\frac{n}{2}, \quad m_{4}+2 m_{5}=\left|F_{4}^{1}\right|+\left|F_{5}^{1}\right|, \quad \text { and } \quad\left|F_{4}^{1}\right|+\left|F_{5}^{1}\right|+2 f_{0} \leq f .
$$

Using $\sqrt{5 / 3}<\frac{4}{3}$ we obtain

$$
\begin{aligned}
\left|\Phi\left(F_{1}, \ldots, F_{5}\right)\right| & \leq\left|\Phi\left(F_{1}^{0}, \ldots, F_{5}^{0}\right)\right|\left|\Phi\left(B, B, B, F_{4}^{1}, F_{5}^{1}\right)\right| \\
& \leq 5^{4 \gamma n} 2^{f_{0}} 3^{\frac{n}{2}-m_{4}-m_{5}} 4^{m_{4}} 5^{m_{5}} \\
& \leq 5^{4 \gamma n} 2^{f_{0}} 3^{\frac{n}{2}}\left(\frac{4}{3}\right)^{f-2 f_{0}} \\
& \leq 5^{4 \gamma n}\left(\frac{9}{8}\right)^{f_{0}} 3^{\frac{n}{2}}\left(\frac{4}{3}\right)^{f} .
\end{aligned}
$$

This easily implies that $F_{4}$ or $F_{5}$ must have size larger than $\frac{3}{8} n+\gamma n$. Indeed, otherwise $f \leq$ $\frac{3}{4} n+2 \gamma n$ and (2) of Claim 23.1 implies that either $F_{4}$ or $F_{5}$ has size larger than $\frac{n}{3}+\gamma n$. By (2) of Theorem [5] and Lemma 13 one can then make, say, $F_{4}$ sum-free by removing at most $\gamma n$ elements. This shows that $f_{0} \leq\left|F_{4}^{0}\right| \leq \frac{n}{4}+\gamma n$ as the largest sum-free set in $B^{0}$ has size at most $\left|B_{0}\right| / 2=n / 4$. Plugging these bounds for $f$ and $f_{0}$ into (11), however, yields $\left|\Phi\left(F_{1}, \ldots, F_{5}\right)\right|<2.22^{n}$ for $G$ with sufficiently large order. This contradicts the lower bound, hence, we can assume that $F_{4}$ has size larger than $\frac{3}{8} n+\gamma n$.

Finally assume that $\left|F_{4}\right|,\left|F_{5}\right| \leq \frac{2}{5} n+\gamma n$. Then $f \leq \frac{4}{5}+2 \gamma n$ and by using (3) of Theorem 5 with Lemma 13 and Lemma 17 we further conclude that there exist sum-free sets $S_{4}$ and $L_{4}$ with $S_{4} \subset L_{4}$ such that $\left|F_{4} \backslash S_{4}\right| \leq \gamma n$ and $\left|L_{4} \cap B\right|=\left|L_{4} \backslash B\right|=\frac{n}{5}$. This implies $f_{0} \leq$ $\left|F_{4}^{0}\right| \leq\left|L_{4} \cap B^{0}\right|+\gamma n \leq \frac{n}{5}+\gamma n$ and we obtain from (11) for $G$ of sufficiently large order that $\left|\Phi\left(F_{1}, \ldots, F_{5}\right)\right|<2.233^{n}$. This again contradicts the lower bound and we conclude that $F_{4}$ is good and so is $F_{5}$ as shown in the beginning. This finishes the proof of the lemma.

\section{EXACT RESUlts}

In this section we prove Theorem 3 and Theorem 4 based on Theorem 17, 9 and 10.

Proof of Theorem 3 and proof of Theorem 4 in case that $G$ has a unique largest sum-free set. We show the proof of Theorem 3 for $r=3$ only, the proof for $r=2$ and that of Theorem 4 in case that $G$ has a unique largest sum-free set follow the same line.

Let $G$ be a type $\mathrm{I}(q)$ group of sufficiently large order $n$ and suppose that $A \subset G$ maximizes the number of sum-free 3-colorings. Then $\kappa_{3}(A) \geq 3^{\mu(G)}$ as this bound is achieved by any largest sum-free set in $G$. Hence, we have $|A| \geq \mu(G)$. Moreover, with $\varepsilon=\frac{1}{80(q+1)}$ Theorem 7 implies that there exists a largest sum-free set $B$ such that $|A \backslash B|<\varepsilon n$.

Assume that there is an $x \in A \backslash B$ otherwise $A=B$ and the theorem follows. If $|G|$ is odd then by Lemma 16 there are at least $\frac{n}{2 q}-1$ pairs $\{a, b\} \in\left(\begin{array}{c}B \\ 2\end{array}\right)$ such that $x=a+b$. If $|G|$ is even 
then by Lemma 18 (with $t=1$ ) for each $a \in B$ there exists $b \in B$ such that $x=a+b$, where possibly $a=b$. If $\varphi(x)=i \in[3]$ then $\varphi$ can be extended to at most 8 sum-free colorings of $\{a, b\}$ if $a \neq b$ and $a, b \in A$, to at most 2 if $a=b, a \in A$ (hence $4<8$ ways for two such pairs $a=b$ and $a^{\prime}=b^{\prime}$ ), and to at most 3 if $a \notin A$ or $b \notin A$. Using $|A \backslash B|<\varepsilon n$ and $\left(1-\frac{1}{2} \log _{3} 8\right)>\frac{1}{20}$ we conclude therefore

$$
\kappa_{3}(A)<3^{\varepsilon n} \cdot 8^{\frac{n}{2 q}} \cdot 3^{\left(|A|-\frac{n}{q}+\varepsilon n+2\right)}<3^{\mu(G)+4 \varepsilon n-\frac{n}{20 q}}=3^{\mu(G)} .
$$

This contradicts the lower bound. Thus, $A=B$ and the theorem follows.

Proof of Theorem 4. It is left to prove Theorem 4 in the case that $G$ has at least two largest sum-free sets. The proofs are quite involved but similar for $r=4$ and for $r=5$. Therefore we show the case $r=5$ only.

Suppose that $A \subset G$ maximizes the number of sum-free 5-colorings. Let $\gamma=3(\log n)^{-1 / 27}$ and let $\mathcal{F}$ be a container family of $A$ as in Theorem 5 . If $A$ maximizes the number of sumfree 5-colorings, then $\left|\kappa_{5}(A)\right| \geq 6^{n / 2}$ due to Proposition 8 , Let $\left(F_{1}, \ldots, F_{5}\right)$ be the quintuple maximizing $\left|\Phi\left(F_{1}^{\prime}, \ldots, F_{5}^{\prime}\right)\right|$ over all $\left(F_{1}^{\prime}, \ldots, F_{5}^{\prime}\right) \in \mathcal{F}^{5}$. From (11) of Observation 6 we infer $6^{n / 2} \leq \kappa_{5}(A) \leq|\mathcal{F}|^{5}\left|\Phi\left(F_{1}, \ldots, F_{5}\right)\right|$ and (1) of Theorem 5 implies

$$
\log _{6}\left|\Phi\left(F_{1}, \ldots, F_{5}\right)\right| \geq \frac{n}{2}-2 n(\log n)^{-1 / 18} .
$$

Hence, we are in the position to apply Theorem 10 to $\left(F_{1}, \ldots, F_{5}\right)$ which entails that there are largest sum-free sets $B_{1}, B_{2}, B_{3}$ in $G$ which satisfy the following.

(1) $B_{3}=B_{1} \triangle B_{2}$ and there is a function $f:[5] \rightarrow[3]$ such that $\left|F_{j} \backslash B_{f(j)}\right|<\gamma n$ for all $j \in[5]$ and $\mathcal{B}=\left(B_{f(1)}, \ldots, B_{f(5)}\right)$ consists of one 4-atom, and two 3-atoms, or

(2) there are four distinct largest sum-free sets $B_{4}, \ldots, B_{7}$ contained in $B_{1} \cup B_{2} \cup B_{3}$ and a function $f:[5] \rightarrow[7]$ such that $\left|F_{j} \backslash B_{f(j)}\right|<\gamma n$ for all $j \in[5]$ and $\mathcal{B}=\left(B_{f(1)}, \ldots, B_{f(5)}\right)$ consists of two 2 -atoms, four 3 -atoms, and one 4 -atom.

Claim 23.2. If (11) applies then $A \subseteq B_{1} \cup B_{2}$ and if (2) applies then we have $A \subseteq B_{1} \cup B_{2} \cup B_{3}$.

Proof. The argument is similar to the one in the proof of Theorem 3, i.e., we show that the existence of an $x \in A \backslash\left(B_{1} \cup B_{2}\right)$ or $x \in A \backslash\left(B_{1} \cup B_{2} \cup B_{3}\right)$, respectively, gives a substantial restriction on how the elements of $A$ may be colored. This then leads to a contradiction to the bound on $\kappa_{5}(A)$. As the proofs of these two cases are similar, we show the second case only.

Assume that there is an $x \in A \backslash\left(B_{1} \cup B_{2} \cup B_{3}\right)$ of which there are at most $5 \gamma n$. As $B_{f(i)} \in$ $\left\{B_{1}, B_{2}, B_{3}\right\}$ for all $i \in[5]$ the atoms of $\mathcal{B}$ and of $\mathcal{B}^{\prime}=\left(B_{1}, B_{2}, B_{3}\right)$ are the same and each has size $n / 8$ due to Corollary 21. Suppose $\varphi(x)=j \in[5]$ so that $x \in F_{j}$ and let $\mathcal{B}(\varepsilon)$ be a 3-atom contained in $B_{f(j)}$. This indeed exists as $B_{f(j)}$ consists of four atoms and $\mathcal{B}$ consists of seven non-zero atoms, four of which are 3 -atoms. By applying Lemma 18 to $\mathcal{B}^{\prime}$ we infer that for each $a \in \mathcal{B}(\varepsilon)$ there is a $b \in \mathcal{B}(\varepsilon)$ such that $x=a+b$. Hence $\varphi$ can be extended to at most 8 sum-free colorings of $\{a, b\}$ if $a \neq b$ and $a, b \in F_{j}$, to at most 2 if $a=b, a \in F_{j}$ (hence $4<8$ ways for two 
such pairs $a=b$ and $a^{\prime}=b^{\prime}$ ), and at most 3 if $a \notin F_{j}$ or $b \notin F_{j}$. Together with Observation 6 and the atom structure of $\mathcal{B}$ this yields

$$
\left|\Phi\left(F_{1}, \ldots, F_{5}\right)\right| \leq 5^{5 \gamma n} \cdot 2^{n / 4} \cdot 3^{3 n / 8} \cdot 4^{n / 8} \cdot 8^{n / 16}=5^{5 \gamma n} \cdot 6^{n / 2} \cdot\left(\frac{\sqrt{8}}{3}\right)^{n / 8},
$$

which is a contradiction to (12).

We next establish that $B_{1} \cup B_{2} \subset A$ in the first case and $B_{1} \cup B_{2} \cup B_{3} \subset A$ in the second case. The following distinction of the colorings will be crucial for this purpose.

Definition 24. A coloring $\varphi \in \Phi_{5}(A)$ is called good if for each $i \in[5]$ we have $\varphi^{-1}(i) \subset L_{i}$ for some largest sum-free set $L_{i}$ of $G$, and

- the tuple $\mathcal{L}=\left(L_{1}, \ldots, L_{5}\right)$ consists of one 4 -atom and two 3-atoms, or

- the tuple $\mathcal{L}=\left(L_{1}, \ldots, L_{5}\right)$ consists of two 2-atoms, four 3-atoms and one 4-atom.

Otherwise $\varphi$ is called a bad coloring.

Note that Definition 24 distinguishes the two cases (11) and (2) as the number of atoms in $\left(L_{1}, \ldots, L_{5}\right)$ is three in the first case and seven in the second case.

Assume for contradiction that $x \in\left(B_{1} \cup B_{2}\right) \backslash A$ or $x \in\left(B_{1} \cup B_{2} \cup B_{3}\right) \backslash A$, respectively. Crucial about the definition is that any good coloring $\varphi$ of $A$ can be extended to at least two sum-free 5-colorings of $A \cup\{x\}$. Indeed, as $x$ belongs to some $k$-atom $\mathcal{L}(\varepsilon), \varepsilon \in\{0,1\}^{5}$, of $\mathcal{L}=\left(L_{1}, \ldots, L_{5}\right)$ for $k \geq 2$, the coloring $\varphi$ can be extended to colorings of $A \cup\{x\}$ by assigning to $x$ one of the $k$ colors associated with $\mathcal{L}(\varepsilon)$. As $\varphi^{-1}(i) \subset L_{i}$ for all $i \in[5]$ these extensions are sum-free. Therefore, assuming the existence of $x$ implies

$$
\left|\Phi_{5}(A \cup\{x\})\right| \geq 2 \mid\left\{\varphi \in \Phi_{5}(A): \varphi \text { is } \operatorname{good}\right\} \mid .
$$

As good and bad colorings of $A$ partition $\Phi_{5}(A)$ it is sufficient to show the following.

Claim 24.1. For $n$ sufficiently large

$$
\mid\left\{\varphi \in \Phi_{5}(A): \varphi \text { is } \operatorname{good}\right\}\left|>6^{n / 2} 2^{-24 \gamma n}>\left(3 \cdot 2^{7 / 2}+\frac{1}{50}\right)^{n / 4}>\right|\left\{\varphi \in \Phi_{5}(A): \varphi \text { is bad }\right\} \mid .
$$

In particular, if (1) holds then $B_{1} \cup B_{2} \subseteq A$ and if (2) holds then $B_{1} \cup B_{2} \cup B_{3} \subseteq A$.

Proof. As the proofs for the two cases are similar we present the proof for the second case only, i.e., when $A \subset B_{1} \cup B_{2} \cup B_{3}$. Recall that $\left|F_{i} \backslash B_{f(i)}\right|<\gamma n$ and $\mathcal{B}=\left(B_{f(1)}, \ldots, B_{f(5)}\right)$ consists of two 2 -atoms, four 3 -atoms and one 4 -atom, each of which has size $n / 8$. We deduce that $\sum_{i \in[5]}\left|B_{f(i)} \backslash F_{i}\right|<12 \gamma n$ since the following contradiction to (12) would arise otherwise:

$$
6^{n / 2-2 n(\log n)^{-1 / 18}} \leq\left|\Phi\left(F_{1}, \ldots, F_{5}\right)\right|<5^{5 \gamma n} 2^{n / 4-12 \gamma n} 3^{n / 2} 4^{n / 8}<6^{n / 2-\gamma n} .
$$

Due to the atom structure of $\mathcal{B}$ note that $\varphi \in \Phi_{5}(A)$ is good if $\varphi^{-1}(i) \subset F_{i} \cap B_{f(i)}$ holds for all $i \in[5]$. Hence

$$
\mid\left\{\varphi \in \Phi_{5}(A): \varphi \text { is good }\right\} \mid>2^{n / 4} 3^{n / 2} 4^{n / 8-12 \gamma n}=6^{n / 2} 2^{-24 \gamma n} .
$$


We now turn to bound the number of bad colorings of $A$. Suppose that $\left(C_{1}, \ldots, C_{5}\right) \in \mathcal{F}^{5}$ maximizes $\mid\left\{\varphi \in \Phi\left(F_{1}^{\prime}, \ldots, F_{5}^{\prime}\right): \varphi\right.$ is bad $\} \mid$ over all $\left(F_{1}^{\prime}, \ldots, F_{5}^{\prime}\right) \in \mathcal{F}^{5}$. By (11) of Observation 6 and $\log _{2}|\mathcal{F}| \leq n(\log n)^{-1 / 18}$ due to (1) of Theorem 5 we have

$$
\begin{aligned}
\mid\left\{\varphi \in \Phi_{5}(A): \varphi \text { is } \operatorname{bad}\right\} \mid & \leq \sum_{\left(F_{1}, \ldots, F_{5}\right) \in \mathcal{F}^{5}} \mid\left\{\varphi \in \Phi\left(F_{1}, \ldots, F_{5}\right): \varphi \text { is bad }\right\} \mid \\
& \leq 2^{5 n(\log n)^{-1 / 18}} \mid\left\{\varphi \in \Phi\left(C_{1}, \ldots, C_{5}\right): \varphi \text { is bad }\right\} \mid .
\end{aligned}
$$

Therefore suppose that $\left|\Phi\left(C_{1}, \ldots, C_{5}\right)\right| \geq\left(3 \cdot 2^{7 / 2}+\frac{1}{100}\right)^{n / 4}$ otherwise the claim follows. Theorem 10 then applies and there are largest sum-free sets $L_{1}, L_{2}, L_{3}$ and a $g:$ [5] $\rightarrow$ [3] or $g:[5] \rightarrow[7]$ such that (11) or (2) of Theorem 10 holds with $F_{i}, B_{i}, f, \mathcal{B}$ replaced by $C_{i}, L_{i}, g$ and $\mathcal{L}=\left(L_{g(1)}, \ldots, L_{g(5)}\right)$. As $C_{1} \cup \cdots \cup C_{5}=A=F_{1} \cup \cdots \cup F_{5}$, the sets $L_{1}, L_{2}, L_{3}$ must be contained in $B_{1} \cup B_{2} \cup B_{3}$ (i.e., $\left.L_{i} \in\left\{B_{1}, \ldots, B_{7}\right\}\right)$ and no $L_{i}, i \in$ [3], is contained in the union of the other two. Hence, case (2) of Theorem 10 applies to $\left(C_{1}, \ldots, C_{5}\right)$ and $\mathcal{L}$ consists of two 2-atoms, four 3 -atoms and one 4-atom. In particular, a $\varphi \in \Phi\left(C_{1}, \ldots, C_{5}\right)$ satisfying $\varphi^{-1}(i) \subset L_{g(i)}$ for all $i \in[5]$ is a good coloring and a bad $\varphi \in \Phi\left(C_{1}, \ldots, C_{5}\right)$ must therefore exhibit a $k \in[5]$ and an $x \in C_{k} \backslash L_{g(k)}$ such that $\varphi(x)=k$. Call $(x, k)$ a bad pair and for such pair let $\Phi\left(C_{1}, \ldots, C_{5} \mid x \mapsto k\right)$ be the set of all (bad) $\varphi \in \Phi\left(C_{1}, \ldots, C_{5}\right)$ with $\varphi(x)=k$. Suppose $(y, \ell)$ maximizes $\left|\Phi\left(C_{1}, \ldots, C_{5} \mid x \mapsto k\right)\right|$ over all bad pairs $(x, k)$, then an argument as in the proof of Claim 23.2 will show that $\left|\Phi\left(C_{1}, \ldots, C_{5} \mid y \mapsto \ell\right)\right|$ is small.

Indeed, fix a 3-atom $\mathcal{L}(\varepsilon)$ in $L_{g(\ell)}$ which exists since $L_{g(\ell)}$ consists of four atoms and $\mathcal{L}$ consists of seven atoms, four of which are 3-atoms. By Lemma 18 for each $a \in \mathcal{L}(\varepsilon)$ there is a $b \in \mathcal{L}(\varepsilon)$ such that $y=a+b$. Coloring $y$ with $\ell$ therefore extends to at most 8 sum-free colorings of $\{a, b\}$ if $a \neq b$ and $a, b \in C_{\ell}$, to at most 2 if $a=b, a \in C_{\ell}$ (hence $4<8$ ways for two such pairs $a=b$ and $a^{\prime}=b^{\prime}$ ), and to at most 3 if $a \notin C_{\ell}$ or $b \notin C_{\ell}$. Hence, as $\left|C_{i} \backslash L_{g(i)}\right|<\gamma n, i \in[5]$, we have

$$
\begin{aligned}
\mid\left\{\varphi \in \Phi\left(C_{1}, \ldots, C_{5}\right): \varphi \text { is bad }\right\} \mid & \leq 5 \gamma n \cdot\left|\Phi\left(C_{1}, \ldots, C_{5} \mid y \mapsto \ell\right)\right| \\
& \leq 5 \gamma n \cdot 5^{5 \gamma n} \cdot 2^{n / 4} \cdot 3^{3 n / 8} \cdot 4^{n / 8} \cdot 8^{n / 16} \\
& =5 \gamma n \cdot 5^{5 \gamma n} \cdot 6^{n / 2} \cdot\left(\frac{\sqrt{8}}{3}\right)^{n / 8}<5.93^{n / 2}<\left(3 \cdot 2^{7 / 2}+\frac{1}{100}\right)^{n / 4}
\end{aligned}
$$

for $n$ sufficiently large. In view of (15) the claim follows.

We have established that either $A=B_{1} \cup B_{2}$ for an independent $\left(B_{1}, B_{2}\right)$ or $A=B_{1} \cup B_{2} \cup B_{3}$ for an independent $\left(B_{1}, B_{2}, B_{3}\right)$. Moreover, the proof shows that the number of colorings in $\Phi_{5}(A)$ is largely dominated by the good colorings.

Call a quintuple $\mathcal{D}=\left(D_{1}, \ldots, D_{5}\right)$ of largest sum-free sets in $A$ substantial if $\mathcal{D}$ consists of 2-atoms, four 3-atoms and one 4-atom. By definition a coloring $\varphi \in \Phi_{5}(A)$ is good if and only if $\varphi \in \Phi(\mathcal{D})$ for some substantial $\mathcal{D}$. Further, if $\mathcal{D}$ and $\mathcal{D}^{\prime}$ are two distinct substantial quintuple, then $\left|\Phi(\mathcal{D}) \cap \Phi\left(\mathcal{D}^{\prime}\right)\right|=o(|\Phi(\mathcal{D})|)$, i.e., most of the good colorings of $A$ are assigned to a substantial $\mathcal{D}$ in a unique way. Indeed, a $\varphi \in \Phi(\mathcal{D}) \backslash \Phi\left(\mathcal{D}^{\prime}\right)$ if for every $k$-atom $\mathcal{D}(\varepsilon)$, 
$k \in\{2,3,4\}$, all $k$ colors are represented in $\mathcal{D}(\varepsilon)$ under $\varphi$, i.e., $|\varphi(\mathcal{D}(\varepsilon))|=k$. It is easily seen that most $\varphi \in \Phi(\mathcal{D})$ satisfy this property.

Finally note that a substantial tuple $\left(D_{1}, \ldots, D_{5}\right)$ in $A=B_{1} \cup B_{2}$ are exactly those having $B_{1}, B_{2}$ and $B_{3}$ as members, two of them twice. There are $\left(\begin{array}{l}3 \\ 2\end{array}\right)\left(\begin{array}{l}5 \\ 2\end{array}\right) 3=90$ many ways to choose such a tuple. Similarly, a substantial tuple $\left(D_{1}, \ldots, D_{5}\right)$ in $A=B_{1} \cup B_{2} \cup B_{3}$ must contain

an independent triple, say $\mathcal{B}^{\prime}=\left(B_{1}^{\prime}, B_{2}^{\prime}, B_{3}^{\prime}\right)$, for which there are $\left(\begin{array}{l}7 \\ 2\end{array}\right) \cdot 4$ many choices. These either extend to a quintuple consisting of pairwise distinct largest sum-free sets, all of which are substantial, or to a quintuple in which exactly one member is repeated, from which all but those containing $\mathcal{B}^{\prime}(1,1,1)$ are substantial. No other tuple is substantial. Together this yields $\left(\begin{array}{l}7 \\ 2\end{array}\right) \cdot 4\left(4 \cdot 3 \cdot 5 !+3 \cdot 4 \cdot \frac{5 !}{2}\right)=181440$ ways to choose a substantial tuple in $A=B_{1} \cup B_{2} \cup B_{3}$. This finishes the proof.

\section{Concluding Remarks}

In the case that $G$ is an even order abelian group the proof of Theorem 4 consists of two parts, a stability and the exact part. Both parts can be extended to more colors provided $G$ contains sufficiently many largest sum-free sets. Indeed, for $r=4,5$ the stability results, Theorem 9 and 10. follow from Lemma 22 and 23. The short argument in Lemma 22 extends to $r=6$ without change using the lower bound $\kappa_{6, G} \geq 2^{3 n / 4} 3^{n / 2}$ (see the footnote in page 14). For $r=7$ we have the lower bound $\kappa_{7, G} \geq \kappa_{7}\left(B_{1} \cup B_{2} \cup B_{3}\right) \geq 4^{7 n / 8}$ obtained by an independent triple $\left(B_{1}, B_{2}, B_{3}\right)$ of largest sum-free sets in $G$. The same argument as in Lemma 22 then implies that for $\left|\Phi\left(F_{1}, \ldots, F_{7}\right)\right| \geq 3.99^{7 n / 8}$ to hold at least six of the seven sets from $\left(F_{1}, \ldots, F_{7}\right)$ must be good and it can then be easily argued that the last $F_{i}$ must be good as well. The second ingredient for the stability result is Lemma 23 which identifies the tuples $\mathcal{B}=\left(B_{1}, \ldots, B_{r}\right)$ maximizing $\left|\Phi\left(\mathcal{B}^{\prime}\right)\right|$ over all tuple $\mathcal{B}^{\prime}$ of largest sum-free sets. This lemma can be extended to $r=6,7$ along the same line. Alternatively, as Corollary 21 reduces the problem in Lemma 23 for an arbitrary $G$ to a related one in $\mathbb{F}_{2}^{t}$ for $t \leq r$, the problem might also be solved by computer search, if needed.

It can be verified that for $r=6$ the optimal structure is obtained for $t=3$ and $\mathcal{B}$ consisting of four 3-atoms and three 4 -atoms, and for $r=7$ it is obtained for $t=3$ and $\mathcal{B}$ consisting of seven 4-atoms. The optimal structure for an arbitrary $r$ is unknown.

Given the stability result the exact configuration can then be derived using the argument given in Section 5 which works for all fixed $r$. Together, we obtain the following for $r=6,7$.

Theorem 25. Let $r \in\{6,7\}$ and let $G$ be an abelian group of sufficiently large even order. Then $\kappa_{r}(A)=\kappa_{r, G}$ if and only if $A=B_{1} \cup B_{2} \cup B_{3}$ for an independent triple $\left(B_{1}, B_{2}, B_{3}\right)$ of largest sum-free sets, provided such exists in $G$.

It would be interesting to extend the results concerning even order groups to arbitrary $r$ and from even order groups to arbitrary type I $(q)$ groups (for $r \geq 4$ ). The methods presented here might be pushed further to give an answer to certain cases of $r>7$ and even order groups. 
They might also be adapted give an answer to particular cases of type $\mathrm{I}(q)$ groups and $r \geq 4$. However, new ideas are needed to solve these problems in general.

\section{REFERENCES}

[1] N. Alon, J. Balogh, P. Keevash, and B. Sudakov. The number of edge colorings with no monochromatic cliques. J. Lond. Math. Soc., II. Ser., 70(2):273-288, 2004.

[2] N. Alon, J. Balogh, R. Morris, and W. Samotij. Counting sum-free sets in Abelian Groups. Israel Journal of Mathematics, 199(1):309-344, 2014.

[3] R. Balasubramanian, G. Prakash, and D.S. Ramana. Sum-free subsets of finite abelian groups of type III. European Journal of Combinatorics, 58:181 - 202, 2016.

[4] J. Balogh, R. Morris, and W. Samotij. Independent sets in hypergraphs. J. Am. Math. Soc., 28(3):669-709, 2015.

[5] P. Diananda and H. Yap. Maximal sum-free sets of elements of finite groups. Proceedings of the Japan Academy, 45(1):1-5, 1969.

[6] P. Erdős. Old and new problems in combinatorial analysis and graph theory. In Second International Conference on Combinatorial Mathematics (New York, 1978), volume 319 of Ann. New York Acad. Sci., pages 177-187. New York Acad. Sci., New York, 1979.

[7] P. Erdős. Some of my favourite problems in various branches of combinatorics. Matematiche (Catania), 47(2):231-240 (1993), 1992. Combinatorics 92 (Catania, 1992).

[8] B. Green. A Szemerédi-type regularity lemma in abelian groups, with applications. Geom. Funct. Anal., 15(2):340-376, 2005.

[9] B. Green and I. Ruzsa. Sum-free sets in abelian groups. Israel Journal of Mathematics, 147(1):157-188, 2005.

[10] C. Hoppen, Y. Kohayakawa, and H. Lefmann. Hypergraphs with many Kneser colorings. European J. Combin., 33(5):816-843, 2012.

[11] H. Lefmann and Y. Person. Exact results on the number of restricted edge colorings for some families of linear hypergraphs. J. Graph Theory, 73(1):1-31, 2013.

[12] H. Lefmann, Y. Person, V. Rödl, and M. Schacht. On colourings of hypergraphs without monochromatic Fano planes. Comb. Probab. Comput., 18(5):803-818, 2009.

[13] H. Lefmann, Y. Person, and M. Schacht. A structural result for hypergraphs with many restricted edge colorings. J. Comb., 1(3-4):441-475, 2010.

[14] O. Pikhurko and Z. B. Yilma. The maximum number of $K_{3}$-free and $K_{4}$-free edge 4 -colorings. J. Lond. Math. Soc., II. Ser., 85(3):593-615, 2012.

[15] A. H. Rhemtulla and A. P. Street. Maximal sum-free sets in finite abelian groups. Bull. Austral. Math. Soc., 2:289-297, 1970.

[16] D. Saxton and A. Thomason. Hypergraph containers. Invent. Math., 201(3):925-992, 2015.

[17] I. Schur. Über die Kongruenz $x^{m}+y^{m}=z^{m}(\bmod p)$. Jahresber. Deutsch. Math.-Verei., 25:114-117, 1916.

[18] H. P. Yap. Maximal sum-free sets in finite abelian groups. IV. Nanta Math., 5(3):70-75, 1972.

[19] H. P. Yap. Maximal sum-free sets in finite abelian groups. V. Bull. Austral. Math. Soc., 13(3):337-342, 1975.

[20] R. Yuster. The number of edge colorings with no monochromatic triangle. J. Graph Theory, 21(4):441-452, 1996.

Departamento de Matemática y Ciencia de la Computación, Universidad de Santiago de Chile E-mail address: han.hiep@gmail.com

CimfaV, Facultad de Ingeniería, Universidad de Valparaíso

E-mail address: andrea.jimenez@uv.cl 